\title{
Learning, Structural Instability and Present Value Calculations
}

\author{
Hashem Pesaran, Davide Pettenuzzo \\ and Allan Timmermann
}

January 2006

CWPE 0602

Not to be quoted without permission 


\title{
Learning, Structural Instability and Present Value Calculations*
}

\author{
Hashem Pesaran \\ University of Cambridge and USC University of Bocconi and Bates and White \\ Allan Timmermann \\ University of California San Diego
}

January 2006

\begin{abstract}
Present value calculations require predictions of cash flows both at near and distant future points in time. Such predictions are generally surrounded by considerable uncertainty and may critically depend on assumptions about parameter values as well as the form and stability of the data generating process underlying the cash flows. This paper presents new theoretical results for the existence of the infinite sum of discounted expected future values under uncertainty about the parameters characterizing the growth rate of the cash flow process. Furthermore, we explore the consequences for present values of relaxing the stability assumption in a way that allows for past and future breaks to the underlying cash flow process. We find that such breaks can lead to considerable changes in present values.
\end{abstract}

JEL Classifications: C11, G12, G22

Keywords: present value, stock prices, structural breaks, Bayesian learning

${ }^{*}$ We would like to thank Ron Smith and Martin Weitzman for their comments on a preliminary version of this paper. 


\section{Introduction}

Present value relations play a key role in economics and finance and are used in testing the permanent income hypothesis, in standard inventory models and to calculate the present value of assets such as stocks and bonds. Computing present values requires forecasting a stream of future values of the variable of interest at horizons that can be long, but finite (as in the case of bonds) or even infinitely long (as in the case of stocks). It is customary in such calculations to assume that the underlying driving process follows a simple ARMA process with stable and known parameter values. This assumption is a gross oversimplification in almost any realistic economic context and so it becomes important both to dispense with the assumption of known parameters and to consider the possibility of past and future breaks in the data generating process of the driving variable.

As an example of a present value relationship, we study in this paper long-lived assets such as stocks. Stocks are claims on unknown future dividends and so the stock price at any point in time must reflect the present value of the expected future dividends. A key question that investors are faced with is therefore how to compute expected values of future dividends in the presence of the considerable uncertainty surrounding not just dividends in the near future but dividends at very distant future points in time. In particular, how high is the growth rate of future dividends likely to be and how much does it vary through time? These are key issues that investors must answer when pricing long-lived assets with unknown future payoffs.

We shall consider the problem of present value calculations under a variety of circumstances. To begin with we assume that the process of the driving variable is known with stable parameters but consider the implications of incomplete learning and parameter uncertainty that arises when dividends or incomes are predicted into an infinite future from a finite past. In the case of geometric random walks with normally distributed innovations we show that expected present value can be divergent even if the parameter uncertainty is confined to the mean of the dividend process. This is a new finding and differs from similar results by Geweke (2001) and Weitzmann (2005) who show that the expected utility does not exist in the case of power utility functions where the consumption growth is normally distributed but with unknown mean and variance. In their set up expected utility is well defined when consumption growth is normally distributed with a known innovation variance. The non-convergence of the discounted sum of expected future values arises because parameter uncertainty increases at a faster rate than the discounting of future outcomes.

In practice, dividends or labour income processes are unlikely to remain stable and may be subject to structural breaks. Indeed, empirical studies have increasingly found evidence of incomplete learning and instability in a range of macroeconomic and financial time series processes that are likely to be related to the determinants of asset payoffs. Stock and Watson (1996) document evidence of breaks in the univariate time-series representation of a wide variety of financial and macroeconomic variables. ${ }^{1}$ Similarly, Clements and Hendry $(1998,1999)$ emphasize the importance

\footnotetext{
${ }^{1}$ Other studies finding evidence of breaks in such time series include Alogoskoufis and Smith (1991), Banerjee et al (1992), Garcia and Perron (1996), Koop and Potter (2004a,b), Pastor and Stambaugh (2001), Paye and Timmermann (2005), Pesaran and Timmermann (2002), Pesaran, Pettenuzzo and Timmermann (2005) and Timmermann (2001).
} 
of breaks to forecasting performance.

Such evidence opens up the possibility that the dividend process underlying common stock portfolios is also subject to breaks, a point verified by Timmermann (2001). Our paper presents new evidence of breaks in US dividends over the period 1872 - 2003. We find evidence of five breaks, three of which cluster over the period 1911-1930 (before the Great Depression), with the other two occurring in 1952 (around the Korean War), and in 1960 (the start of the Golden Age). The parameter estimates in the associated regimes differ significantly both in economic and statistical terms in a way that suggests that the dividend process has become less volatile but also more persistent through time.

Building on this evidence, we next explore how to forecast future dividends and compute the present value of dividends in the context of a model where the dividend growth process is subject to occasional structural breaks. Such breaks give rise to considerable uncertainty about the stock price when compared to a model that ignores breaks although the latter, as we argue in this paper, is clearly mis-specified. Our analysis uses the hierarchical hidden Markov chain model introduced in Pesaran, Pettenuzzo and Timmermann (2005) for the purpose of forecasting time-series that are subject to multiple breaks. Building on work by Chib (1998), this approach introduces a meta distribution that characterizes the distribution from which parameters within each dividend growth regime are drawn following a new break. Without this approach, forecasting future values of dividends is infeasible unless, of course, the possibility of future breaks to the parameters of the dividend process is ruled out. Using Gibbs sampling techniques we draw values from the parameter distribution within the regime that is in effect at the time of the forecast. To allow for possible breaks, we next draw new values of the discrete state indicator that characterizes how future states evolve. In the event that a future break occurs, new values of the parameters of the subsequent regime are drawn from the meta distribution.

Using the parameter estimates for the break point process fitted to US dividends, we find that the present value stock price is very sensitive to the underlying modeling assumptions for the dividend process. In particular, it depends on whether the possibility of past breaks during the historical sample is considered and also whether future breaks are allowed for. Since the regimes identified for the dividend process are typically quite persistent, there is no particular ranking of the present value stock price computed under no (historical or future) breaks, under historical breaks only or under past and future break scenarios. Instead, the ranking will reflect the value of the dividend growth rate in the current state relative to its historical average computed across different regimes.

The outline of the paper is as follows. Section 2 presents new theoretical results on the existence of present values under parameter estimation uncertainty. Section 3 discusses the role of structural breaks and presents empirical results for a model with multiple break points fitted to US dividend data. Section 4 shows how the present value stock price can be computed under different assumptions concerning parameter instability and reports empirical results for US data. Finally, Section 5 concludes. 


\section{Parameter Uncertainty and Present Value Calculations}

Many intertemporal optimization problems result in rational expectations models with future expectations whose solution involves calculation of the discounted value of an infinite sum of forecasts formed at time $T$, for many periods ahead into the future. A simple example is given by

$$
y_{T}=\lim _{H \rightarrow \infty}\left\{\sum_{h=1}^{H} \delta^{h} E\left(x_{T+h} \mid \mathcal{I}_{T}\right)\right\},
$$

where $\mathcal{I}_{T}$ is the forecaster's information at time $T, \quad r>0$ is the (known) discount rate so the discount factor, $\delta=(1+r)^{-1}$, lies in $(0,1)$, and $E\left(x_{T+h} \mid \mathcal{I}_{T}\right)$ is the conditional expectation taken with respect to the probability distribution(s) assumed for the driving process, $\left\{x_{t}\right\}$, over the past $m$ periods $(t=T-m+1, T-m+2, \ldots, T)$, and the future $(t=T+1, T+2, . ., T+h)$, where $m$ is the length of the estimation window while $h$ is the forecast horizon. In general, the driving process need not be known or stable. In many applications in finance and economics it is assumed that $\left\{x_{t}\right\}$ follows the geometric random walk model

$$
\Delta \ln x_{t+1}=\mu+\sigma \varepsilon_{t+1}
$$

where $\mu$ and $\sigma$ are fixed constants, and $\varepsilon_{t+1}$ is identically and independently distributed with zero means and unit variances. For given (known) values of $\mu$ and $\sigma$, and assuming that these values apply to the past as well as to the indefinite future, we have

$$
E\left(x_{T+h} \mid \mathcal{I}_{T} ; \mu, \sigma\right)=\left(e^{h \mu}\right)\left[M_{\varepsilon}(\sigma)\right]^{h},
$$

where $M_{\varepsilon}(\sigma)$ is the moment generating function of $\varepsilon_{t}$, assuming that it exists. Under the above assumptions the present value, $y_{T}$, is convergent and is given by

$$
y_{T}=\frac{\delta e^{\mu} M_{\varepsilon}(\sigma) x_{T}}{1-\delta e^{\mu} M_{\varepsilon}(\sigma)}
$$

so long as $\lambda=\delta e^{\mu} M_{\varepsilon}(\sigma)<1$. In the case of normally distributed errors $M_{\varepsilon}(\sigma)$ exists and is given by $\exp \left(0.5 \sigma^{2}\right)$. This yields the familiar result in the literature, $\lambda=\delta \exp \left(\mu+0.5 \sigma^{2}\right) .^{2}$

\subsection{Unknown $\mu$ with a Known $\sigma^{2}$}

Consider next the case where $\sigma$ is known but $\mu$ is unknown and estimated based on the past observations, $\mathbf{X}_{m, T}=\left(x_{T-m+1}, x_{T-m+2}, \ldots, x_{T}\right)^{\prime}$, with a Gaussian prior:

$$
\mu \sim N\left(\underline{\mu}, \underline{\sigma}_{\mu}^{2}\right), \underline{\sigma}_{\mu}^{2}>0
$$

Assuming that $\varepsilon_{t+1} \sim N(0,1)$, the posterior distribution of $\mu$ will also be Gaussian and is given by

$$
\mu \mid \mathbf{X}_{m, T}, \sigma, \underline{\mu}, \underline{\sigma}_{\mu}^{2} \sim N\left(\bar{\mu}, \bar{\sigma}_{\mu}^{2}\right)
$$

\footnotetext{
${ }^{2}$ Notice, however, that even when $\mu$ and $\sigma$ are known, the present value calculations are quite fragile in the case of geometric random walk models, where non-convergent outcomes will follow if the innovations, $\varepsilon_{t}$, are distributed as a Student-t. A similar result has also been pointed out by Geweke (2001) in the case of expected utility optimization where the consumption growth follows a geometric random walk model and the utility function is of the CRRA variety.
} 
where (see, for example, Geweke (2005, pp. 25-27))

$$
\begin{aligned}
\bar{\mu} & =\bar{\sigma}_{\mu}^{2}\left(\frac{\underline{\mu}}{\underline{\sigma}_{\mu}^{2}}+\frac{m \bar{x}_{m, T}}{\sigma^{2}}\right), \\
\bar{x}_{m, T}=m^{-1} \sum_{t=T-m+1}^{T} x_{t}, \text { and } & \\
\bar{\sigma}_{\mu}^{2} & =\left(\frac{1}{\underline{\sigma}_{\mu}^{2}}+\frac{m}{\sigma^{2}}\right)^{-1}
\end{aligned}
$$

In this case

$$
\begin{aligned}
E\left(x_{T+h} \mid \mathcal{I}_{T} ; \sigma, \underline{\mu}, \underline{\sigma}_{\mu}^{2}\right) & =x_{T}\left[E\left(e^{h \mu} \mid \mathcal{I}_{T} ; \sigma, \underline{\mu}, \underline{\sigma}_{\mu}^{2}\right)\right]\left[M_{\varepsilon}(\sigma)\right]^{h} \\
& =x_{T} E\left(e^{h \mu} \mid \mathcal{I}_{T} ; \sigma, \underline{\mu}, \underline{\sigma}_{\mu}^{2}\right) e^{\frac{1}{2} h \sigma^{2}}
\end{aligned}
$$

where

$$
E\left(e^{h \mu} \mid \mathcal{I}_{T} ; \sigma, \underline{\mu}, \underline{\sigma}_{\mu}^{2}\right)=e^{h \bar{\mu}+\frac{1}{2} h^{2} \bar{\sigma}_{\mu}^{2}},
$$

and the individual elements in the infinite sum, (1), exist and are given by

$$
E\left(x_{T+h} \mid \mathcal{I}_{T} ; \sigma\right)=x_{T} e^{h \bar{\mu}+\frac{1}{2} h^{2} \bar{\sigma}_{\mu}^{2}+\frac{1}{2} h \sigma^{2}}=x_{T}\left(e^{\bar{\mu}+\frac{1}{2} h \bar{\sigma}_{\mu}^{2}+\frac{1}{2} \sigma^{2}}\right)^{h} .
$$

Finally, the present value, truncated at forecast horizon $H$, becomes

$$
y_{T: T+H}=x_{T} \sum_{h=1}^{H}\left(\delta e^{\bar{\mu}+\frac{1}{2} h \bar{\sigma}_{\mu}^{2}+\frac{1}{2} \sigma^{2}}\right)^{h}=x_{T} \sum_{h=1}^{H}[\rho(h, m)]^{h},
$$

where

$$
\rho(h, m)=e^{-\ln (1+r)+\bar{\mu}+\frac{1}{2} h \bar{\sigma}_{\mu}^{2}+\frac{1}{2} \sigma^{2}} .
$$

To check if this is convergent as $H \rightarrow \infty$, we first note that

$$
\begin{gathered}
\bar{\mu}=\frac{\bar{x}_{m, T}+\left(\frac{\sigma^{2}}{m}\right)\left(\frac{\underline{\mu}}{\underline{\sigma}_{\mu}^{2}}\right)}{1+\left(\frac{\sigma^{2}}{m}\right)\left(\frac{1}{\underline{\sigma}_{\mu}^{2}}\right)}=\bar{x}_{m, T}\left[1-\left(\frac{\sigma^{2}}{m}\right)\left(\frac{1}{\underline{\sigma}_{\mu}^{2}}\right)\right]+\left(\frac{\sigma^{2}}{m}\right)\left(\frac{\underline{\mu}}{\underline{\sigma}_{\mu}^{2}}\right)+O\left(\frac{1}{m^{2}}\right), \\
\bar{\sigma}_{\mu}^{2}=\frac{\sigma^{2}}{m}\left(\frac{1}{1+\frac{1}{m}\left(\frac{\sigma^{2}}{\sigma_{\mu}^{2}}\right)}\right)=\frac{\sigma^{2}}{m}+O\left(\frac{1}{m^{2}}\right) .
\end{gathered}
$$

Using these results in (11) yields $\rho(h, m)=\delta e^{\frac{1}{2} \sigma^{2}} e^{g(h, m)}$, where

$$
g(h, m)=\bar{x}_{m, T}\left[1-\left(\frac{\sigma^{2}}{m}\right)\left(\frac{1}{\underline{\sigma}_{\mu}^{2}}\right)\right]+\left(\frac{\sigma^{2}}{m}\right)\left(\frac{\underline{\mu}}{\underline{\sigma}_{\mu}^{2}}\right)+\frac{\sigma^{2}}{2}\left(\frac{h}{m}\right)+O\left(\frac{h}{m^{2}}\right),
$$

or after some algebra

$$
g(h, m)=\bar{x}_{m, T}+\left(\frac{\sigma^{2}}{m}\right)\left[\frac{\underline{\mu}-\bar{x}_{m, T}}{\underline{\sigma}_{\mu}^{2}}\right]+\frac{\sigma^{2}}{2}\left(\frac{h}{m}\right)+O\left(\frac{h}{m^{2}}\right) .
$$


Typically one expects $\left(\underline{\mu}-\bar{x}_{m, T}\right) /\left(m \underline{\sigma}_{\mu}^{2}\right)$ to be quite small and the present value would be dominated by the term $\frac{\sigma^{2}}{2}\left(\frac{h}{m}\right)$. Therefore,

$$
y_{T: T+H} \approx x_{T} \sum_{h=1}^{H}\left(e^{-\ln (1+r)+\frac{1}{2} \sigma^{2}(1+h / m)+\bar{x}_{m, T}}\right)^{h},
$$

and even if $\hat{\lambda}_{m, T}=\exp \left(-\ln (1+r)+\bar{x}_{m, T}+0.5 \sigma^{2}\right)<1$ (the estimated certainty equivalence convergence condition), $y_{T: T+H}$ will be divergent as $H \rightarrow \infty .^{3}$

The only case where the present value convergences in the presence of the estimation uncertainty will be if the limit of $h / m$ as $h \rightarrow \infty$ is bounded from above by some constant, $\kappa<$ $2\left[\bar{x}_{m, T}-\ln (1+r)\right] / \sigma^{2}-1$. This corresponds to a thought experiment in which the length of the estimation sample $(m)$ somehow grows at a sufficiently fast rate with the forecast horizon, $h$, so that $\lim (h / m)$ tends to $\kappa<2[\mu-\ln (1+r)] / \sigma^{2}-1$, as $h$ and $m$ tend to infinity jointly. In practice, of course, such thought experiments have little relevance since the estimation window (often determined by the historical data set available) and the forecast horizon are separate concepts and there is no reason why they should be linked in any particular way. Furthermore, in cases with evidence of instability in the parameters of the dividend process, an unbiased estimator of the parameters of the dividend generating process can only be obtained by restricting the estimation sample to the post-break data. This provides another reason for why $m$ is finite in practice. In the following we shall, without loss of generality, assume $m=T$ and suppress the $m$ notation for simplicity.

\subsection{Unknown $\mu$ and $\sigma^{2}$}

The non-convergence problem of the present value will be accentuated if we also assume that $\sigma$ is unknown and is estimated from the past data, $\mathbf{X}_{m, T}$. For example, using conjugate priors for $\mu$ and $\sigma^{2}$ the posterior distribution of $\mu$ will be $t$-distributed and $E\left(e^{h \mu} \mid \mathcal{I}_{T} ; \underline{\mu}, \underline{\sigma}_{\mu}^{2}, \underline{\sigma}^{2}, \underline{v}\right)$ ceases to exist for any $h>0$, where $\underline{\sigma}^{2}$ and $\underline{v}$ are the parameters of the gamma prior density assumed for $\sigma^{2}$ which can be written conveniently as

$$
\frac{\underline{\sigma}^{2}}{\sigma^{2}} \mid \underline{\sigma}^{2}, \underline{v} \sim \chi^{2}(\underline{v}) .
$$

As pointed out by Geweke (2001), the use of non-conjugate priors for $\mu$ and $\sigma^{2}$ does help in resolving the non-existence of $E\left(e^{h \mu} \mid \mathcal{I}_{T} ; \underline{\mu}, \underline{\sigma}_{\mu}^{2}, \underline{\sigma}^{2}, \underline{v}\right)$. However, it does not resolve the non-convergence of the infinite sums that are involved in present value calculations.

Non-Bayesian approaches to dealing with the uncertainty of $\mu$ and $\sigma^{2}$ are unlikely to help either. One possible approach would be to bootstrap the present values. This involves (i) drawing $\mu^{(b)}$ and $\sigma^{(b)}$ from the observed empirical distribution of the estimators of $\mu$ and $\sigma^{2}$ (say $\hat{\mu}_{m, T}$ and $\hat{\sigma}_{m, T}^{2}$ ), (ii) computing present values for each choice of $\mu^{(b)}$ and $\sigma^{(b)}$ denoted as $y_{T}^{(b)}$, and (iii) obtaining the bootstrap present value as $B^{-1} \Sigma_{b=1}^{B} y_{T}^{(b)}$, where $B$ is the total number of bootstraps. However, for this procedure to yield a convergent outcome it will be required that $y_{T}^{(b)}$ is convergent for each

\footnotetext{
${ }^{3}$ The above analysis also shows the danger of letting $m \rightarrow \infty$ first before computing the limit of the present value with $H \rightarrow \infty$. In reality $m$ could be quite large but still finite as $H \rightarrow \infty$.
} 
$b$, which is extremely unlikely unless the empirical distribution of $\hat{\mu}_{m, T}$ and $\hat{\sigma}_{m, T}^{2}$ is constrained so that all draws from that distribution satisfy the conditions $\delta e^{\mu^{(b)}} M_{\varepsilon}\left(\sigma^{(b)}\right)<1$ for all $b$.

\subsection{Trend Stationary Log-linear Driving Processes}

The non-convergence problem continues to be present if the unit root process in (2) is replaced by the following trend stationary process:

$$
\Delta\left[\ln x_{t+1}-a-\mu(t+1)\right]=-(1-\rho)\left(\ln x_{t}-a-\mu t\right)+\sigma \varepsilon_{t+1},
$$

where $|\rho|<1$, and as before $\mu$ represents the average growth of the logarithm of the driving process, $x_{t}$. In the case of this process

$$
\ln \left(x_{T+h} / x_{T}\right)=-\left(1-\rho^{h}\right)\left(\ln x_{T}-a-\mu T\right)+\mu h+\sigma \sum_{j=1}^{h} \rho^{j} \varepsilon_{T+j},
$$

and

$$
E\left(x_{T+h} \mid \mathcal{I}_{T} ; a, \mu, \rho, \sigma\right)=e^{-\left(1-\rho^{h}\right)\left(\ln x_{T}-a-\mu T\right)}\left(e^{h \mu}\right)\left[\prod_{j=1}^{h} M_{\varepsilon}\left(\sigma \rho^{j}\right)\right],
$$

which is a direct generalization of (8) and reduces to it for $\rho=1$. It is clear that the various issues discussed for the unit root case readily apply here. Even if $\varepsilon_{t+1}$ has a moment generating function, the present value is unlikely to exist if $\mu$ is not known with certainty. For example, suppose $a, \rho$ and $\sigma$ are known and $\mu$ is estimated based on the regression of $\ln x_{t}-\rho \ln x_{t-1}-a(1-\rho)$ on $(1-\rho) t+\rho$. Assuming, as before, that conditional on $a, \rho$ and $\sigma$ the prior probability distribution of $\mu$ is Gaussian and given by (5), then the posterior distribution of $\mu$ will be given by

$$
\mu \mid \mathbf{X}_{T}, a, \rho, \sigma, \underline{\mu}, \underline{\sigma}_{\mu}^{2} \sim N\left(\bar{\mu}, \bar{\sigma}_{\mu}^{2}\right)
$$

where

$$
\begin{gathered}
\bar{\mu}=\bar{\sigma}_{\mu}^{2}\left(\frac{\underline{\mu}}{\underline{\sigma}_{\mu}^{2}}+\frac{\hat{\mu}_{T}}{\hat{\sigma}_{T}^{2}}\right), \\
\hat{\mu}_{T}=\frac{\sum_{t=1}^{T}\left[\ln x_{t}-\rho \ln x_{t-1}-a(1-\rho)\right][(1-\rho) t+\rho]}{\sum_{t=1}^{T}[(1-\rho) t+\rho]^{2}}, \\
\hat{\sigma}_{T}^{2}=\frac{\sigma^{2}}{\sum_{t=1}^{T}[(1-\rho) t+\rho]^{2}}, \text { and } \bar{\sigma}_{\mu}^{2}=\left(\frac{1}{\underline{\underline{\alpha}}_{\mu}^{2}}+\frac{1}{\hat{\sigma}_{T}^{2}}\right)^{-1} .
\end{gathered}
$$

Hence

$$
\begin{aligned}
E\left(x_{T+h} \mid \mathcal{I}_{T} ; a, \rho, \sigma\right) & =\left[e^{-\left(1-\rho^{h}\right)\left(\ln x_{T}-a\right)} \prod_{j=1}^{h} M_{\varepsilon}\left(\sigma \rho^{j}\right)\right] E_{\mu}\left(e^{\left[h+\left(1-\rho^{h}\right) T\right] \mu}\right) \\
& =\left[e^{-\left(1-\rho^{h}\right)\left(\ln x_{T}-a\right)} \prod_{j=1}^{h} M_{\varepsilon}\left(\sigma \rho^{j}\right)\right] e^{\left[h+\left(1-\rho^{h}\right) T\right] \bar{\mu}+\frac{1}{2}\left[h+\left(1-\rho^{h}\right) T\right]^{2} \bar{\sigma}_{\mu}^{2}},
\end{aligned}
$$


and for a fixed $T$ its rate of expansion is governed by the term $\exp \left(.5 \bar{\sigma}_{\mu}^{2} h^{2}\right)$. Therefore, as far as the existence of the present value is concerned the outcomes are very similar irrespective of whether the logarithm of the driving variable has a unit root or is trend stationary. The differences between the two cases is a matter of degree and differs only due to the differences in the precision with which $\mu$ is estimated under the two cases. Under the unit root process the precision of $\mu$ is of order $T^{-1}$, while when $\ln x_{t}$ is trend stationary it is given by $T^{-3 / 2}$.

\subsection{Present Values with a Stochastic Discount Factor: The Lucas Tree Model}

Normally the discount rate is formed as the risk-free rate plus some risk-premium to reflect the uncertain nature of future payoffs and correct for correlations between dividend shocks and variations in the stochastic discount factor. It is clearly of interest to relate the discount rate-taken to be fixed and strictly exogenous so far-to the growth rate in dividends, using equilibrium consumption based asset pricing models. In the case of consumption based asset pricing models the expression for the present value is more complicated and depends on a stochastic discount factor that varies with $x$. In the context of a representative agent model with the utility function, $u\left(c_{t}\right)$, we have ${ }^{4}$

$$
y_{T}=\lim _{H \rightarrow \infty}\left\{\sum_{h=1}^{H} \delta^{h} E\left(\frac{u^{\prime}\left(c_{t+h}\right)}{u^{\prime}\left(c_{t}\right)} x_{T+h} \mid \mathcal{I}_{T}\right)\right\} \text {, }
$$

which reduces to the present value expression (1) in the risk neutral case where $u(c)$ is linear. But for a general specification of $u(c)$ the analysis of convergence of the present value depends on the form of the utility function and the nature of the dependence of $c_{t+h}$ and $x_{t+h}$ in a general equilibrium context. Although such a general analysis is beyond the scope of the present paper, certain analytical results can be obtained for the Lucas's tree model (Lucas, 1978) where consumption is equal to dividends $\left(c_{T+h}=x_{T+h}\right)$ and the utility function is specified to have the power form $u(c)=$ $(1-\gamma)^{-1}\left(c^{1-\gamma}-1\right)(\gamma \neq 1)$. In this case,

$$
y_{T}=\lim _{H \rightarrow \infty}\left\{\sum_{h=1}^{H} E\left(e^{-h \ln (1+r)+(1-\gamma)\left(\ln x_{T+h}-\ln x_{T}\right)} \mid \mathcal{I}_{T}\right)\right\},
$$

and under the geometric random walk model (2) with a known mean and variance we have

$$
E\left(y_{T} \mid \mu, \sigma^{2}\right)=x_{T} \lim _{H \rightarrow \infty}\left\{\sum_{h=1}^{H} E\left(e^{-h \ln (1+r)+(1-\gamma) \mu h+0.5(1-\gamma)^{2} \sigma^{2} h} \mid \mu, \sigma^{2}, \mathcal{I}_{T}\right)\right\}
$$

which is convergent for given values of $\mu$ and $\sigma^{2}$ so long as $-\ln (1+r)+(1-\gamma) \mu+0.5(1-\gamma)^{2} \sigma^{2}<0$.

\footnotetext{
${ }^{4}$ See, for example, Cochrane (2005, p.24). Deriving (16) from the first order inter-temporal optimization conditions also requires that the transversality condition

$$
\lim _{h \rightarrow \infty} E\left(\frac{u^{\prime}\left(c_{T+h}\right)}{u^{\prime}\left(c_{T}\right)} y_{T+h} \mid \mathcal{I}_{T}\right)=0
$$

is satisfied.
} 
Consider now the case where $\mu$ is unknown and continue to assume that $\sigma^{2}$ is given (known). Then using the above results we have

$$
E\left(e^{(1-\gamma) \mu h+0.5(1-\gamma)^{2} \sigma^{2} h} \mid \sigma^{2}, \mathcal{I}_{T}\right)=e^{(1-\gamma) \bar{\mu} h+0.5(1-\gamma)^{2} \sigma^{2} h+0.5(1-\gamma)^{2} h^{2} \bar{\sigma}_{\mu}^{2}}
$$

where $\bar{\mu}$ and $\bar{\sigma}_{\mu}^{2}$ are the posterior mean and variance of $\mu$ given by (6) and (7) where $m=T$ and that do not vary with $h$. Hence, the elements in the series expansion of $E\left(y_{T} / x_{T} \mid \sigma^{2}\right)$ eventually will be dominated by terms $e^{0.5(1-\gamma)^{2} h^{2} \bar{\sigma}_{\mu}^{2}}, h=1,2, \ldots$ and the present value expression will be divergent unless $\gamma=1$, corresponding to the special case of log-utility.

\subsection{Possible Solutions to the Non-Convergence Problem}

The reason for the fragility of the present value under the geometric random walk model with constant but unknown parameters can be illustrated using a simple discrete state process for $\mu$. Suppose that over the forecast horizon $T+1, T+2, \ldots, T+H, \mu$ can take any one of the values $\mu_{1}, \mu_{2}, \ldots, \mu_{m}$ with probabilities $\pi_{1}, \pi_{2}, \ldots, \pi_{m}$ where $\sum_{i=1}^{m} \pi_{i}=1$, and $1>\pi_{i}>0$. To simplify the analysis also assume that $\sigma^{2}, \mu_{i}$ and $\pi_{i}$ are known at time $T$. Under this example, the present value is given by

$$
y_{T}=x_{T}\left\{\sum_{i=1}^{m} \pi_{i} \lim _{H \rightarrow \infty} \sum_{h=1}^{H} e^{-h \ln (1+r)+h \mu_{i}}\left[M_{\varepsilon}(\sigma)\right]^{h}\right\} .
$$

Since $1>\pi_{i}>0, y_{T}$ exists if $\delta e^{\mu_{i}} M_{\varepsilon}(\sigma)<1$ for all $i$. Contrast this result with the associated certainty equivalent expression that accounts for uncertainty about the value of the underlying mean parameter (but disregards uncertainty about future dividend innovations):

$$
y_{T}^{C E}=x_{T}\left\{\lim _{H \rightarrow \infty} \sum_{h=1}^{H} e^{-h \ln (1+r)+h \bar{\mu}_{\pi}}\left[M_{\varepsilon}(\sigma)\right]^{h}\right\},
$$

where $\bar{\mu}_{\pi}=\sum_{i=1}^{m} \pi_{i} \mu_{i}$. The condition for $y_{T}^{C E}$ to exist is given by $\delta e^{\bar{\mu}_{\pi}} M_{\varepsilon}(\sigma)<1$. Clearly, it is possible for the latter to be satisfied without $\delta e^{\mu_{i}} M_{\varepsilon}(\sigma)<1$ being satisfied for all $i$. A sufficiently large $\mu_{i}$, even if it is extremely unlikely (with $\pi_{i}$ very close to zero), can result in divergence of $y_{T}$, although for all other outcomes that are much more likely the associated infinite sums could be convergent.

It is clear from this example that the non-convergence of the present value arises from the particular combinations of (i) a geometric random walk driving process, (ii) an infinite horizon and (iii) constant, but unknown parameters drawn from a Gaussian posterior distribution. One could consider relaxing any one or all of these elements. We discuss the first two assumptions below and then deal with the parameter stability assumption in more detail in the next section.

\subsubsection{Use of Linear Driving Processes}

The non-convergence problem can be avoided altogether if the geometric random walk model is abandoned in favour of a linear driving process. Consider for example the simple random walk 
model with drift

$$
x_{t}=\mu+x_{t-1}+\sigma \varepsilon_{t} .
$$

Then $E\left(x_{T+h} \mid \mathcal{I}_{T}\right)=x_{T}+\mu h$, and

$$
\begin{aligned}
y_{T} & =\lim _{H \rightarrow \infty}\left\{\sum_{h=1}^{H} \delta^{h} E\left(x_{T+h} \mid \mathcal{I}_{T}\right)\right\} \\
& =\frac{x_{T}}{1-\delta}+\mu \sum_{\tau=1}^{\infty} h \delta^{h}
\end{aligned}
$$

or

$$
y_{T}=\frac{x_{T}}{1-\delta}+\frac{\mu \delta}{(1-\delta)^{2}}
$$

Uncertainty surrounding $\mu$ can easily be dealt with in a way that does not cause non-convergence problems. Generalizing the process to higher order models with possible serial correlation in the

innovations, $\varepsilon_{t}$, would not alter the main conclusion. For example, for the $p^{t h}$ order driving process

$$
x_{t}=\left(1-\Sigma_{j=1}^{p} \rho_{j}\right) \mu+\Sigma_{j=1}^{p} \rho_{j} x_{t-j}+\sigma \varepsilon_{t},
$$

conditional on $\boldsymbol{\rho}=\left(\rho_{1}, \rho_{2}, \ldots, \rho_{p}\right)^{\prime}$ we have

$$
y_{T} \mid \boldsymbol{\rho}=\frac{\Sigma_{j=0}^{p-1} \phi_{j}(\delta) x_{T-j}}{1-\phi_{0}(\delta)}+\frac{\delta \mu\left(1-\Sigma_{j=1}^{p} \rho_{j}\right)}{(1-\delta)\left(1-\phi_{0}(\delta)\right)}
$$

where

$$
\phi_{j}(\delta)=\Sigma_{i=j+1}^{p} \rho_{i} \delta^{i-j}, \text { for } j=0,1, \ldots, p-1 .
$$

Under parameter uncertainty

$$
y_{T}=\Sigma_{j=0}^{p-1} E\left[\frac{\phi_{j}(\delta)}{1-\phi_{0}(\delta)} \mid \mathbf{X}_{T}\right] x_{T-j}+\frac{\delta}{1-\delta} E\left(\frac{\mu\left(1-\Sigma_{j=1}^{p} \rho_{j}\right)}{1-\phi_{0}(\delta)} \mid \mathbf{X}_{T}\right)
$$

where expectations are taken with respect to the posterior distribution of $\mu$ and $\boldsymbol{\rho}$. These expectations are likely to exist for a sufficiently large $T$, and do not depend on the forecast horizon.

The problem with this approach is, as pointed out by Campbell, Lo and MacKinlay (1997, p. 258), that linear models for real dividends, consumption or labour income do not fit the data well since these series tend to grow exponentially over time. This means that linear models are usually dominated by log-linear specifications.

\subsubsection{Use of Finite Horizons}

A simple, but rather ad hoc, solution would be to define the present values over a given finite future, say $\tilde{H}$, and then write the solution as

$$
y_{T}(\tilde{H})=\sum_{h=1}^{\tilde{H}} \delta^{h} E\left(x_{T+h} \mid \mathcal{I}_{T}\right)
$$


which is finite so long as $E\left(x_{T+h} \mid \mathcal{I}_{T}\right)$ exists for $h=1, \ldots, \tilde{H}$. In practice the choice of $\tilde{H}$ could be problematic. In the case of the life cycle consumption model, $\tilde{H}$ can be viewed as the life of the household and treated as a truncated random variable. For example, we could assume that (for $0<\theta<1)$

$$
\begin{aligned}
\operatorname{Pr}(\tilde{H} & =s)=\frac{(1-\theta) \theta^{s}}{\theta\left(1-\theta^{\bar{H}}\right)}, \text { for } s=1,2, \ldots, \bar{H} \\
& =0, \text { for } s>\bar{H},
\end{aligned}
$$

where $\bar{H}$ is an arbitrarily large but finite value. The non-truncated case where $\bar{H} \rightarrow \infty$ yields the familiar geometric distribution used by Yaari (1965), Cass and Yaari (1967) and Blanchard and Fischer (1989) to model uncertain life times in models of household consumption. Integrating out the uncertainty of $\tilde{H}$ we have

$$
E_{\tilde{H}}\left[y_{T}(\tilde{H})\right]=\frac{(1-\theta)}{\theta\left(1-\theta^{\bar{H}}\right)} \sum_{s=1}^{\bar{H}} \sum_{h=1}^{s} \theta^{s} \delta^{h} E\left(x_{T+h} \mid \mathcal{I}_{T}\right),
$$

which can be written more compactly as

$$
E_{\tilde{H}}\left[y_{T}(\tilde{H})\right]=\sum_{s=1}^{\bar{H}} \frac{1-\theta^{\bar{H}-s+1}}{\theta\left(1-\theta^{\bar{H}}\right)}(\theta \delta)^{s} E\left(x_{T+s} \mid \mathcal{I}_{T}\right) .
$$

In this set up the choice of $\bar{H}$ is of secondary importance. However, it is worth noting that for $\bar{H} \rightarrow \infty$ the uncertain life time present value problem reduces to

$$
\lim _{\tilde{H} \rightarrow \infty} E_{\tilde{H}}\left[y_{T}(\tilde{H})\right]=\theta^{-1} \sum_{s=1}^{\infty}(\theta \delta)^{s} E\left(x_{T+s} \mid \mathcal{I}_{T}\right),
$$

which is the infinite horizon problem with a lower discount factor given by $\theta \delta$. By increasing the discount rate the stochastic life time assumption will help towards achieving convergence, but does not resolve the problem altogether. A finite $\bar{H}$ would still be required in general.

\section{Present Value Models with Structural Breaks}

Perhaps a more appealing way to handle the non-convergence problem is to relax the assumption that the parameters of the underlying growth process are constant through time. This assumption clearly goes to the root of the non-convergence: As long as there is even an infinitesimal probability of drawing a set of parameters for which the (constant) growth rate exceeds the discount rate, the present value will not exist. Conversely, if the parameters of the growth rate are subject to structural breaks, there are cases where the growth rate temporarily exceeds the discount rate, yet the present value continues to exist. This happens provided that the underlying driving process most of the time grows at a slower rate than the discount rate. The condition for the existence of the present value is now the rather weaker one that paths leading to an unbounded present value 
have zero probability. This will trivially hold if the distribution of the maximum growth rate is truncated so that it always falls below the discount rate, but can also hold in the absence of this assumption.

Breaks to the cash flow process will not, however, in and of itself, resolve the problem. This can easily be seen as follows. Suppose that the mean of the increment to the logarithm of the first-differenced future dividend process falls in different regimes during the period $T+1, \ldots T+h$. Denote the number of these regimes by $N_{h}$ and let their duration be $h_{1}, \ldots, h_{N_{h}}$, so that $\sum_{i=1}^{N_{h}} h_{i}=h$. Equivalently, the fraction of the time spent in regime $i$ is given by $\pi_{i}=h_{i} / h, 0<\pi_{i}<1$. Suppose that $\mu_{i} \sim N\left(\bar{\mu}, \sigma_{\mu}^{2}\right)$. We then get the present value as follows:

$$
\begin{aligned}
y_{T} & =E_{T}\left[x_{T} \sum_{h=1}^{H} \exp \left[-\ln (1+r) h+h \sum_{i=1}^{N_{h}} \pi_{i} \mu_{i}+\sigma \sum_{j=1}^{h} \varepsilon_{j}\right]\right] \\
& =x_{T} \sum_{h=1}^{H} \exp \left[-\ln (1+r) h+h\left(\bar{\mu}+\frac{1}{2} \sigma^{2}\right)+\frac{1}{2} \sigma_{\mu}^{2} h^{2} \sum_{i=1}^{N_{h}} \pi_{i}^{2}\right] .
\end{aligned}
$$

Notice that the last term is not convergent as $H \rightarrow \infty$. Hence while the possibility of breaks adds some flexibility to the model, one has to be careful to ensure convergence of the present value either by using a finite $H$, by truncating the distribution from which the future growth rate is drawn, or through some other means.

\subsection{A Dividend Model with Breaks}

To illustrate the above issues, we next consider the empirical evidence of breaks in the dividend process underlying US stocks. Real dividends underlying broadly diversified stock market indices are often assumed to follow a simple process of the form (2) with $\varepsilon_{t+1} \sim N(0,1)$. Depending on the frequency at which dividends are modelled, autoregressive dynamics may also be present, in which case the process can be generalized to

$$
\Delta \ln \left(x_{t+1}\right)-\mu=\sum_{i=1}^{p} \beta_{i}\left(\Delta \ln \left(x_{t+1-i}\right)-\mu\right)+\sigma \varepsilon_{t+1},
$$

where $\beta_{i}(i=1, \ldots, p)$ are autoregressive parameters and $\mu$ reflects the long-run mean of the dividend growth rate, whereas $\tilde{\mu}=\mu\left(1-\sum_{i=1}^{p} \beta_{i}\right)$ is the intercept for the $\operatorname{AR}(\mathrm{p})$ process in $\Delta \ln \left(x_{t+1}\right)$. Both specifications (2) and (29) assume that the parameters of the dividend growth process remain constant through time-an assumption that, in view of the significant shocks to economic growth observed throughout the twentieth century, is unlikely to be satisfied over the long sample periods typically used for estimation of the parameters of the dividend growth process, see Timmermann (2001).

To capture the possibility of structural shifts in the parameters of the dividend growth process, we adopt the change-point process proposed by Chib (1998). ${ }^{5}$ This approach assumes that shifts to

\footnotetext{
${ }^{5} \mathrm{McCulloch}$ and Tsay (1993) is another prominent example of breakpoint analysis in a Bayesian setting.
} 
the parameters of a time-series process are determined by the evolution in a discrete state variable, $S_{t}$, initialized so that $S_{1}=1$ and increasing over time to capture the possibility of breaks. Every time the state variable increases by one unit, the parameters of the process shift. Hence, if the break point indicator $S_{T}=K+1$, this means that there were $K$ breaks between time 1 and time $T$. For example, assuming that the dividend process can be characterized as an AR(1) model whose parameters are subject to breaks, we have

$$
\begin{array}{ll}
\Delta \ln \left(x_{t+1}\right)-\mu_{1}=\beta_{1}\left(\Delta \ln \left(x_{t}\right)-\mu_{1}\right)+\sigma_{1} \varepsilon_{t+1}, & \tau_{0} \leq t \leq \tau_{1} \\
\Delta \ln \left(x_{t+1}\right)-\mu_{2}=\beta_{2}\left(\Delta \ln \left(x_{t}\right)-\mu_{2}\right)+\sigma_{2} \varepsilon_{t+1}, & \tau_{1}+1 \leq t \leq \tau_{2} \\
\vdots & \vdots \\
\Delta \ln \left(x_{t+1}\right)-\mu_{K+1}=\beta_{K+1}\left(\Delta \ln \left(x_{t}\right)-\mu_{K+1}\right)+\sigma_{K+1} \varepsilon_{t+1}, & \tau_{K}+1 \leq t \leq T
\end{array}
$$

where $\tau_{1}, \ldots, \tau_{K}$ are the breakpoints and $\left\{\mu_{S_{t+1}}, \beta_{S_{t+1}}, \sigma_{S_{t+1}}^{2}\right\}$ are the parameters associated with the dividend process regime that is in effect at time $t+1$. Our other assumptions follow Pesaran, Pettenuzzo and Timmermann (2005) which we next briefly review. The state variable, $S_{t+1}$, can either remain in the $k^{\text {th }}$ regime, which happens with probability $p_{k k}$ or move on to the next regime, which happens with probability $p_{k, k+1}=1-p_{k k}$. These probabilities are assumed to be drawn independently across regimes from a beta distribution with prior parameters $\underline{a}$ and $\underline{b}$ :

$$
p_{i i} \sim \operatorname{Beta}(\underline{a}, \underline{b}), \text { for } i=1,2 \ldots, K \text {. }
$$

For the $\operatorname{AR}(1)$ specification the parameters determining the conditional mean of the dividend growth process, $\boldsymbol{\beta}_{i}=\left(\tilde{\mu}_{i}, \beta_{i}\right)$ for $i=1,2, \ldots, K+1$, are drawn from a Gaussian distribution, $\boldsymbol{\beta}_{S_{t+1}} \sim N\left(\mathbf{b}_{0}, \mathbf{B}_{0}\right)$, while the error term precision parameters, $\sigma_{S_{t+1}}^{-2}$, are identically, independently distributed (IID) draws from a Gamma distribution, $\sigma_{j}^{-2} \sim G\left(v_{0}, d_{0}\right)$. At the level of the meta distribution, we make the following distributional assumptions:

$$
\begin{aligned}
\mathbf{b}_{0} & \sim N\left(\underline{\boldsymbol{\mu}_{\beta}}, \underline{\boldsymbol{\Sigma}_{\beta}}\right) \\
\mathbf{B}_{0}^{-1} & \sim W\left(\underline{v_{\beta}}, \underline{\mathbf{V}_{\beta}^{-1}}\right),
\end{aligned}
$$

where $W($.$) is a Wishart distribution. \underline{\boldsymbol{\mu}_{\beta}}, \underline{\boldsymbol{\Sigma}_{\beta}}, \underline{v_{\beta}}$ and $\mathbf{V}_{\beta}^{-1}$ are hyperparameters that are specified a priori. Finally, the error term precision, $v_{0}$ and $d_{0} \overline{\text { are }}$ assumed to follow an exponential and Gamma distribution, respectively, with hyperparameters $\underline{\rho_{0}}, \underline{c_{0}}$ and $\underline{d_{0}}$ :

$$
\begin{aligned}
& v_{0} \sim \operatorname{Exp}\left(\underline{\rho_{0}}\right) \\
& d_{0} \sim \operatorname{Gamma}\left(\underline{c_{0}}, \underline{d_{0}}\right) .
\end{aligned}
$$

We refer to this specification as the composite-meta model. All prior parameters are under-scored.

\subsection{Estimation Results}

We use data from Shiller (2000) available at http://www.econ.yale.edu/ $\sim$ shiller/data.htm. This provides monthly dividends paid by the firms included in a broad index of US firms. The data 
runs from 1871:03 to 2003:09. Nominal dividends are divided by the consumer price index to get a time-series of real dividends. Statistics on the first-differenced values of this series are provided in Table 1. Monthly dividend growth rates are serially correlated and left-skewed with fat tails. Figure 1 plots the associated time-series and indicates strong evidence of instability in the underlying process as it has evolved over the sample. Most notably, the volatility was very high in the early parts of the sample, and around World War II, and has become much lower after this period. In addition, the persistence of the series appears to have shifted over time as reflected in a more volatile (less persistent) time-series plot in the early parts of the sample. These are of course only visual impressions and must be confirmed by a more formal econometric analysis.

To this end we next document the presence of breaks in the dividend process. Table 2 shows estimates for a variety of models with different numbers of breaks. Bayes factors based on the ratios of the marginal likelihoods for models with different numbers of breaks suggest selecting a model with five breaks, i.e. a break occurring roughly every 25 years. In fact, assuming equal prior probabilities on the models with between zero and six breaks, almost all of the posterior probability mass goes to the model with five breaks.

Based on the posterior modes for the probability of a shift in the state variable, $S_{t}$, the five breaks are estimated to have occurred in 1911, 1922, 1930 (at the beginning of the Great Depression), 1952 (around the Korean War) and in 1960 (the start of the Golden Age of Capitalism). Figure 2 shows that the date of the first break is very poorly determined with probabilities of a break in individual months well below $6 \%$ and spread out between 1900 and 1920. The remaining break dates are more precisely determined with modal probabilities varying from 0.15 to 0.35 .

Table 3 reports parameter estimates for the model with five breaks (six regimes). As one might expect from a sample period as heterogenous as the twentieth century, there is considerable variation in the parameters across regimes. The intercept parameter varies from -0.02 to 0.16 , while the $\operatorname{AR}(1)$ parameter varies from a low point of 0.37 (between 1871 and 1911) to a high point of 0.73 between 1930 and 1952. Confirming the visual impression from Figure 1, the standard deviation of the dividend process has varied considerably from a peak of 0.78 prior to 1911 to its value of 0.15 after 1960. Clearly the dividend process has become less volatile but also more persistent through time. Finally, the mean value of the 'stayer' probability parameter that characterizes the duration of the various states has varied from a high of 0.9997 in the regime prior to 1911 to 0.985 in the regime over the period 1952 to 1960.

Consistent with the large variation across regimes in the parameters of the dividend growth process, the mean value of the standard errors of the meta distribution parameters $\left(b_{0}(1), b_{0}(2)\right)$ are quite large at 0.04 and 0.08 , respectively. In fact, Table 4 shows that the $95 \%$ confidence interval for $b_{0}(1)$, the parameter in the meta distribution characterizing the mean intercept across regimes, goes from -0.021 to 0.104 and from 0.43 to 0.68 for $b_{0}(2)$, the parameter in the meta distribution characterizing the mean persistence across regimes. Following a future break, the parameters of the dividend process will be drawn from the meta distribution so these values indicate that there is considerable uncertainty about the process driving future dividend growth.

Figure 3 shows that parameter instability of the dividend growth process has a large effect on 
the predictive density. It shows the predictive density under three different models at horizons of 1, 12, 24, 36, 48 and 60 months. The first model is the full-sample, no-break model, while the other two models allow for past breaks and past and future breaks. The model that allows for past breaks effectively bases predictions of future values on the parameters from the last regime-the regime prevailing from 1960-2003 in this case-while the model that allows for future breaks starts off from this regime but lets new parameters following a future break be drawn from the meta distribution. This also explains why the predictive densities are more concentrated under the break regimes in Figure 3 since the standard deviation of innovations to dividends was very low in this regime compared with the full-sample average value. While the dispersions of the densities are quite different, however, in this regime the centering of the dividend growth process is not greatly affected by the presence of breaks.

The relationship between the predictive density under breaks and under no breaks can be quite different depending on the parameter values in the regime from which the forecast is calculated. To see this, we plot in Figure 4 the same three graphs but now for the case where the parameters in the last regime are based on their values in the regime prevailing during 1922-1930. It now becomes clear that, particularly at the longer horizons, the three predictive densities are very different once breaks are considered. In this case breaks shift the mean growth rate to the right compared with the no-break case. The reason why the difference is largest at the longer horizons is due to the cumulated effect of having different mean and persistence parameters under the three scenarios. Since the forecasts from the AR(1) model are computed based on the same initial value of the dividend process, differences in the parameters have a relatively smaller effect at short horizons. This observation is by no means unique to the regime from 1922-1930 and-as shown in Figure 5-also holds for the regime that was in effect from 1952-1960.

Differences between predictive densities under the two breakpoint models can be explained with reference to Figure 6. This figure plots the weight on the current regime (thus assuming that the parameter values for the final regime remain in effect) as a function of the forecast horizon. This is similar to a survival plot for the current state and shows how the probability of a break (computed as one minus the 'stayer' probability plotted in Figure 6) increases to more than $50 \%$ as the forecast horizon extends beyond five years.

\section{Present Value Stock Price under Breaks}

As we showed in Section 2, to compute the present value of future dividends, we need to evaluate an expression of the form

$$
\lim _{H \rightarrow \infty} y_{T}=\lim _{H \rightarrow \infty} \sum_{h=1}^{H} \delta^{h} E_{T}\left[x_{T+h}\right]=\lim _{H \rightarrow \infty} \sum_{h=1}^{H} \exp (-h \ln (1+r)) E_{T}\left[x_{T+h}\right] .
$$

Notice that $E_{T}[$.$] is calculated not just conditional on current dividends, x_{T}$, but on the entire past sequence $\left\{x_{t}\right\}_{t=1}^{T}$. Hence the complete historical track record of dividends matters when forecasting future dividends. 
To compute the future stream of dividends, we use the Gibbs sampler to generate draws from the predictive distribution of $\Delta \ln \left(x_{T+1}\right), \Delta \ln \left(x_{T+2}\right), . ., \Delta \ln \left(x_{T+H}\right)$. To see how this works, consider the sum of $\log$ first-differences of dividends between period $T+1$ and period $T+H$ :

$$
\sum_{h=1}^{H} \Delta \ln \left(x_{T+h}\right)=\ln \left(x_{T+H} / x_{t}\right), \quad(H \geq 1)
$$

so

$$
x_{T+H}=x_{T} \exp \left(\sum_{h=1}^{H} \Delta \ln \left(x_{T+h}\right)\right)
$$

or, in terms of present values,

$$
\delta^{H} x_{T+H}=x_{T} \exp \left(\sum_{h=1}^{H} \Delta \ln \left(x_{T+h}\right)-H \ln (\delta)\right) .
$$

First assume that there are no breaks between period $T$ and $T+h$. When dividends follow the $\mathrm{AR}(1)$ process (30), the value of $\Delta \ln \left(x_{T+h}\right)$ is given by

$$
\Delta \ln \left(x_{T+h}\right)-\mu_{S_{T}}=\beta_{S_{T}}^{h}\left(\Delta \ln \left(x_{T}\right)-\mu_{S_{T}}\right)+\sum_{i=1}^{h} \beta_{S_{T}}^{h-i} \varepsilon_{T+1+h-i} .
$$

Using (37), we see after some algebra that

$$
\ln \left(x_{T+h} / x_{T}\right)=h \mu_{S_{t}}+\frac{\beta_{S_{T}}\left(1-\beta_{S_{T}}^{h}\right)}{1-\beta_{S_{T}}}\left[\Delta \ln \left(x_{T}\right)-\mu_{S_{T}}\right]+\varepsilon_{T+1: T+h},
$$

where $\varepsilon_{T+1: T+h}$ denotes the weighted shocks to the present value of dividends between period $T$ and $T+h$, defined by

$$
\varepsilon_{T+1: T+h}=\varepsilon_{T+h}+\left(\frac{1-\beta_{S_{T}}^{2}}{1-\beta_{S_{T}}}\right) \varepsilon_{T+h-1}+\left(\frac{1-\beta_{S_{T}}^{3}}{1-\beta_{S_{T}}}\right) \varepsilon_{T+h-2} \ldots+\left(\frac{1-\beta_{S_{T}}^{h}}{1-\beta_{S_{T}}}\right) \varepsilon_{T+1} .
$$

It is now easily seen that

$$
\operatorname{Var}\left(\varepsilon_{T+1: T+h}\right)=\frac{h+\frac{\beta_{S_{T}}^{2}\left(1-\beta_{S_{T}}^{2 h}\right)}{1-\beta_{S_{T}}^{2}}-\frac{2 \beta_{S_{T}}\left(1-\beta_{S_{T}}^{h}\right)}{1-\beta_{S_{T}}}}{\left(1-\beta_{S_{T}}\right)^{2}} .
$$

Future dividends can therefore be simulated by drawing a set of parameters, $\left\{\mu_{S_{T},} \beta_{S_{T}}\right\}$, and, for these parameters, compute

$$
x_{T+h}=x_{T} \exp \left\{h \mu_{S_{T}}+\frac{\beta_{S_{T}}\left(1-\beta_{S_{T}}^{h}\right)}{1-\beta_{S_{T}}}\left[\Delta \ln \left(x_{T}\right)-\mu_{S_{T}}\right]+\varepsilon_{T+1: T+h}\right\},
$$

where $\varepsilon_{T+1: T+h}$ has mean zero and variance as given in (41). 
Turning to the case that allows for breaks between periods $T+1$ and $T+h$, and recalling that $\tilde{\mu}_{S_{T+i}}=\mu_{S_{T+i}}\left(1-\beta_{S_{T+i}}\right)$, we have

$$
\begin{aligned}
\Delta \ln \left(x_{T+1}\right)= & \tilde{\mu}_{S_{T+1}}+\beta_{S_{T+1}} \Delta \ln \left(x_{T}\right)+\varepsilon_{T+1} \\
\Delta \ln \left(x_{T+2}\right)= & \tilde{\mu}_{S_{T+2}}+\beta_{S_{T+2}} \tilde{\mu}_{S_{T+1}}+\beta_{S_{T+2}} \beta_{S_{T+1}} \Delta \ln \left(x_{T}\right)+\varepsilon_{T+2}+\beta_{S_{T+2}} \varepsilon_{T+1} \\
\Delta \ln \left(x_{T+3}\right)= & \tilde{\mu}_{S_{T+3}}+\beta_{S_{T+3}} \tilde{\mu}_{S_{T+2}}+\beta_{S_{T+3}} \beta_{S_{T+2}} \tilde{\mu}_{S_{T+1}}+\beta_{S_{T+3}} \beta_{S_{T+2}} \beta_{S_{T+1}} \Delta \ln \left(x_{T}\right) \\
& +\varepsilon_{T+3}+\beta_{S_{T+3}} \varepsilon_{T+2}+\beta_{S_{T+3}} \beta_{S_{T+2}} \varepsilon_{T+1} \\
& \vdots \\
\Delta \ln \left(x_{T+h}\right)= & \tilde{\mu}_{S_{T+h}}+\varepsilon_{T+h}+\sum_{j=1}^{h-1}\left(\prod_{i=j+1}^{h} \beta_{S_{T+1}}\right)\left(\tilde{\mu}_{S_{T+j}}+\varepsilon_{T+j}\right)+\left(\prod_{i=j+1}^{h} \beta_{S_{T+i}}\right) \Delta \ln \left(x_{T}\right) .
\end{aligned}
$$

Comparing this expression to (39), clearly, the presence of breaks complicates calculations of future expected dividends very considerably and numerical methods are required to compute the present value.

\subsection{Computing Present Values}

In practice we calculate the present value of the stock price as follows:

$$
\lim _{H \rightarrow \infty} y_{T}=\lim _{H \rightarrow \infty} \sum_{h=1}^{H} \exp (-h \ln (1+r)) \int x_{T+h} p\left(x_{T+h} \mid \mathcal{I}_{T}\right) d x_{T+h},
$$

where $\mathcal{I}_{t}$ is again the forecaster's information set at time $T$, which we shall assume comprises past dividends only, i.e. $\mathcal{I}_{T}=\left\{x_{1}, \ldots, x_{T}\right\} \cdot p\left(x_{T+h} \mid \mathcal{I}_{T}\right)$ is the predictive density of dividends at time $T+h$ conditional on $\mathcal{I}_{T}$.

The expectation is computed under three different scenarios capturing different assumptions about the forecaster's beliefs:

1. A model that accounts for parameter estimation uncertainty but ignores past and future breaks to dividends by using the predictive density:

$$
p\left(x_{T+h} \mid S_{T+h}=1, \mathcal{I}_{T}\right)=\int p\left(x_{T+h} \mid \Theta_{t}, S_{T+h}=1, \mathcal{I}_{T}\right) \pi\left(\Theta_{T} \mid S_{T+h}=1, \mathcal{I}_{T}\right) d \Theta_{T},
$$

where $\Theta$ are the constant model parameters whose posterior distribution given the data at time $T$ is $\pi\left(\Theta \mid S_{T+h}=1, \mathcal{I}_{T}\right)$.

2. A model that accounts for historical breaks to the dividend process but ignores the possibility of future breaks (and hence assumes that the last regime stays in effect forever but with uncertain parameters) by using the predictive density:

$p\left(x_{T+h} \mid S_{T+h}=K+1, \mathcal{I}_{T}\right)=\int p\left(x_{T+h} \mid \Theta_{K+1}, S_{T+h}=S_{T}=K+1, \mathcal{I}_{T}\right) \pi\left(\Theta_{K+1} \mid \mathcal{H}, p, \mathcal{I}_{T}\right) d \Theta_{K+1}$,

where $\Theta_{K+1}$ are the parameters in the last regime (labelled $K+1$ ), while $\mathcal{H}$ is the set of hyper parameters. 
3. A model that accounts for parameter estimation uncertainty as well as past and future breaks to the dividend process by using the following predictive density:

$$
\int p\left(x_{T+h} \mid S_{T+h}=K+n_{b}+1, S_{T}=K+1, \mathcal{I}_{T}\right)
$$

where $n_{b}$ is the maximum number of out-of-sample breaks so the predictive density can be calculated (integrating out uncertainty about the dates of the breaks, $\tau_{K+1}=T+j_{1}, \ldots, \tau_{K+n_{b}}=$ $\left.j_{n_{b}}\right)$ as

$$
\begin{aligned}
& p\left(x_{T+h} \mid S_{T+h}=K+n_{b}+1, S_{T}=K+1, \mathcal{I}_{T}\right)=\sum_{j_{1}=1}^{h-n_{b}+1} \ldots \sum_{j_{n_{b}}=j_{n_{b}-1}+1}^{h} \int \cdots \int \\
& p\left(x_{T+h}, \Theta_{K+2}, \ldots, \Theta_{K+n_{b}+1}, \mathcal{H}, S_{T+h}=K+n_{b}+1\right. \\
& \left.\tau_{K+1}=T+j_{1}, \ldots, \tau_{K+n_{b}}=T+j_{n_{b}}, S_{T}=K+1, \mathcal{I}_{T}\right) \\
& \times \pi\left(\tau_{K+1}=T+j_{1}, \ldots, \tau_{K+n_{b}}=t+j_{n_{b}} \mid S_{T+h}=K+n_{b}+1, S_{T}=K+1\right) \\
& \times \pi\left(\Theta_{K+2}, \ldots, \Theta_{K+n_{b}+1}, \mathcal{H} \mid \mathcal{I}_{T}\right) d \Theta_{K+2} \ldots d \Theta_{K+n_{b}+1} d \mathcal{H} .
\end{aligned}
$$

To get a more complete picture of the possible impact of breaks on the present value price, we compute the stock price under the three scenarios based on different terminal regimes. One thousand draws from the Gibbs sampler were used (after discarding the first 500 draws) to forecast dividends and the present value of dividends. In computing the monthly dividends, we set the forecast horizon at $H=1000$ and assumed an annualized discount rate of $10 \%$. The parameters of the prior were as follows: $p_{i i} \sim \operatorname{Beta}(\underline{a}, \underline{b})$ with $\underline{a}=\underline{b}=0.5$. We assume an uninformative prior for the parameters of the conditional mean of the dividend process by setting $\boldsymbol{\mu}_{\beta}=\mathbf{0}_{2 \times 1}$, $\mathbf{V}_{\beta}=1000 \mathbf{I}_{2}$ (recall that $\boldsymbol{\beta}_{i}=\left(\tilde{\mu}_{i}, \beta_{i}\right)^{\prime}$ in the $i^{\text {th }}$ regime). The hyperparameters determining the

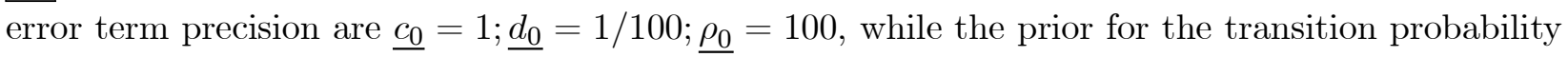
matrix is assumed to be drawn from a $\operatorname{Gamma}\left(\underline{a}_{0}, \underline{b}_{0}\right)$ distribution with $\underline{a}_{0}=1 ; \underline{b}_{0}=1 / 10$.

To shed light on the practical importance of our choice of $H$, the terminal value at which dividends are computed, Figure 6 plots the present value of the expected dividend as a function of the forecast horizon, $H$. The sum of expected discounted dividends stabilizes rapidly under all three models, suggesting that, in this parameterization and for our choice of discount rate $r=10 \%$ per annum, the present value is not very sensitive to our choice of $H$. Our earlier theoretical results suggest, however, that if we were to let $H \rightarrow \infty$, the results could be quite different. Consistent with this, when we chose a smaller value for the discount rate of $r=5 \%$ per annum, the present value series failed to converge, blowing up in the process. These results suggest that in the presence of parameter estimation uncertainty and model instability the present value stock price can be very sensitive to modeling assumptions.

\subsection{Empirical Results}

Table 5 reports the stock price computed under these assumptions relative to the stock price from the model that ignores model instability which we normalize at $100 .{ }^{6}$ As indicated by the parameter

\footnotetext{
${ }^{6}$ Since we are using a finite horizon in these calculations, normalizing the present values in this way is innocuous.
} 
estimates in Table 3, the mean value of the log first-differenced dividends in the regime prevailing during 1922-1930 was unusually high. As a result, in this regime the stock price computed under the assumption that the last regime stays in effect and no future breaks occur (the middle column in Table 5) was nearly $40 \%$ higher than the full-sample, no-break stock price. In this regime, the stock price computed under the composite-meta model that allows for both past and future breaks lies in the middle of the full-sample and current regime values, $20 \%$ above the no-break price. Stock prices under past and/or present breaks that exceed the value under the no-break assumption are also observed under the parameters based on the regime that was in effect during the period 1952-1960.

Conversely, the stock price based on dividend growth parameter values from the regime prevailing from 1930-1952 falls below the full-sample value by $7 \%$ since dividend growth was quite low in this regime as indicated by the negative intercept for this state shown in Table 3. Furthermore, in this regime the stock price computed under the composite meta distribution, at 101, is only marginally above the full-sample value. A similar set of results is obtained on the basis of the parameters from the last regime prevailing during 1960-2003.

The reason for these rankings is easy to understand from Figure 7: At short investment horizons, the weight on the current state tends to be very high, but this weight declines gradually as the horizon is expanded and the weight on draws from the meta distribution increases. Consequently, the stock price under the current regime lies above both the full-sample value and the price computed under the composite meta distribution whenever dividend growth is very high in the current regime, i.e. the parameters associated with the current regime are drawn from the right tail of the meta distribution. In this situation, the stock price under the composite-meta distribution is also likely to be considerably higher than its full-sample counterpart, but it falls below the value conditioned on remaining in the last regime since dividend growth after a future break is likely to be below the growth rate in the current regime.

These results also demonstrate that, in general, the stock price under the composite meta distribution will tend to be above the full-sample value due to the convexity of the mapping from the dividend growth rate to the stock price implied by the present value relation (see Timmermann (2001)). This explains why we see higher stock prices as a result of accounting for parameter uncertainty and model instability. Furthermore, model instability generally increases the effect of parameter uncertainty. The intuition for this finding is that under breaks fewer observations are effectively used to estimate the model parameters in the last regime, so the standard errors of the parameters tend to increase under breaks compared with full-sample estimates.

However, in a given regime, any ranking between stock prices under the three scenarios is in fact possible. For example, if the current state experiences a sufficiently low dividend growth rate and the state is highly persistent, then the effect of conditioning the stock price on the dividend growth parameters from the current state will dominate the convexity effect and hence the stock price under the composite meta distribution (as well as under the assumption that the current state remains in effect) will be smaller than the full-sample, no break price.

Furthermore, stock prices under the model that accounts for breaks need not exceed prices under a no-break assumption in models where shocks to the dividend growth process are correlated 
with a stochastic discount factor and uncertainty surrounding future dividend growth leads to a higher risk premium. This will occur, for example, in consumption asset pricing models where dividends have a large positive correlation with consumption growth. But our discussion of the Lucas model in Section 2.4 suggests that taking account of possible correlations between dividends and consumption growth might not be sufficient to resolve the non-convergence problem so long as there are important uncertainties surrounding the future mean dividend growth rates.

\section{Conclusion}

This paper showed how to compute stock prices as the present value of future dividends when we do not assume that the dividend process is stable through time and that its parameters are known. We showed that stock prices can be quite sensitive to the nature of the assumptions concerning uncertainty and instability of the parameters of the dividend process. These findings suggest that our understanding of the dynamics in stock prices can be improved by focusing on the uncertainty surrounding the underlying fundamentals process.

Our emphasis on the sensitivity of present values to uncertainty about the growth rate in different 'regimes' or states of nature is closely related to the literature on how investors' learning about the dividend growth process can give rise to the 'excess volatility' patterns observed for asset prices, c.f. Timmermann (1993). Even with a finite horizon, present values can be very sensitive to small changes in the estimated growth rate, particularly as this gets close to the discount rate. It is also related to recent work on asset pricing puzzles by Weitzmann (2005) who, following earlier insights by Geweke (2001), points out the sensitivity of equilibrium asset prices and returns to assumptions concerning the precision of the parameters characterizing the distribution from which fundamentals are generated, questions the ergodicity assumption made in much of the rational expectations literature and proposes modifications to this. For example, Weitzmann (2005) writes that "the unobservable nature of structural growth parameters adds to expectation beliefs a permanent thick-tailed background layer of uncertainty that never converges to a stationary-ergodic rational expectations equilibrium."

The empirical results presented here clearly have implications for the equity premium puzzle, although we chose not to address this issue here. Recent papers by Barro (2005), de Santis (2005) and Weitzmann (2005) emphasize the importance of parameter uncertainty, instability and rare events as potential explanations of the historically large equity premium. Indeed, through their large effect on the present value stock price, persistent shifts in the dividend growth rate tend to increase the uncertainty about future returns which may be a reason why a larger equity premium is required compared to the standard model that ignores such effects. ${ }^{7}$

\footnotetext{
${ }^{7}$ Allowing for estimation uncertainty could also be important in resolving the so called Deaton's paradox, namely the excess smoothness of observed consumption growth to changes in labour income growth. See, for example, Deaton (1992).
} 


\section{References}

[1] Alogoskoufis, G.S. and R. Smith, 1991, The Phillips Curve, the Persistence of Inflation, and the Lucas Critique: Evidence from Exchange Rate Regimes. American Economic Review 81, $1254-1275$.

[2] Banerjee, A., R. Lumsdaine and J.H. Stock, 1992, Recursive and Sequential Tests of the UnitRoot and Trend-Break Hypotheses: Theory and International Evidence. Journal of Business and Economic Statistics 10, 271-287.

[3] Barro, R.J., 2005, Rare Events and the Equity Premium. Mimeo, Harvard.

[4] Blanchard, Oliver J. and Stanley Fischer (1989), Lectures on Macroeconomics, MA: MIT Press.

[5] Campbell, J.Y., A. Lo and C. MacKinlay, 1997, The Econometrics of Financial Markets. Princeton University Press: Princeton.

[6] Cass, David and Menahem E. Yaari (1967), "Individual Saving, Aggregate Capital Accumulation, and Efficient Growth." In K. Shell (ed.), Essays on the Theory of Optimal Economic Growth. Cambridge, MA: MIT Press.

[7] Chib, S., 1998, Estimation and Comparison of Multiple Change Point Models, Journal of Econometrics, 86, 221-241.

[8] Clements, M.P. and D.F. Hendry, 1998, Forecasting Economic Time Series, Cambridge University Press.

[9] Clements, M.P. and D.F. Hendry, 1999, Forecasting Non-stationary Economic Time Series, The MIT Press.

[10] Cochrane, J., 2005, Asset Pricing. Revised Edition. Princeton University Press: Princeton.

[11] de Santis, M., 2005, Movements in the Equity Premium: Evidence from Time-Varying VAR. Unpublished manuscript.

[12] Deaton, A., (1992), Understanding Consumption, Clarendon Press, Oxford.

[13] Garcia, R. and P. Perron, 1996, An Analysis of the Real Interest Rate under Regime Shifts. Review of Economics and Statistics 78, 111-125.

[14] Geweke, J., 2001, A Note on Some Limitations of CRRA Utility. Economics Letters 71, 341-346.

[15] Geweke, J., 2005, Contemporary Bayesian Econometrics and Statistics. New York: Wiley.

[16] Koop, G. and S. Potter, 2004a, Forecasting and Estimating Multiple Change-point Models with an Unknown Number of Change-points. Mimeo, University of Leicester and Federal Reserve Bank of New York. 
[17] Koop, G. and S. Potter, 2004b, Prior Elicitation in Multiple Change-point Models. Mimeo, University of Leicester and Federal Reserve Bank of New York.

[18] Lucas, R.E., 1978, Asset Prices in an Exchange Economy. Econometrica 46, 1429-1445.

[19] McCulloch, R.E. and R. Tsay, 1993, Bayesian Inference and Prediction for Mean and Variance Shifts in Autoregressive Time Series. Journal of the American Statistical Association 88, 965978.

[20] Pastor, L. and R.F. Stambaugh, 2001, The Equity Premium and Structural Breaks. Journal of Finance, 56, 1207-1239.

[21] Paye, B. and A. Timmermann, 2005, Instability of Return Prediction Models. Forthcoming in Journal of Empirical Finance.

[22] Pesaran, M.H., D. Pettenuzzo and A. Timmermann, 2005, Forecasting Time Series Subject to Multiple Structural Breaks. Forthcoming in Review of Economic Studies.

[23] Pesaran, M.H. and A. Timmermann, 2002, Market timing and return prediction under model instability. Journal of Empirical Finance, 9, 495-510.

[24] Shiller, R.J., 2000, Irrational Exuberance. Princeton: Princeton University Press.

[25] Stock, J.H. and M.W. Watson, 1996, Evidence on Structural Instability in Macroeconomic Time Series Relations. Journal of Business and Economic Statistics 14, 11-30.

[26] Timmermann, A., 1993, How Learning in Financial Markets Generates Excess Volatility and Predictability in Stock Prices, Quarterly Journal of Economics 108, 1135-1145.

[27] Timmermann, A., 2001, Structural Breaks, Incomplete Information, and Stock Prices. Journal of Business and Economic Statistics 19, 299-315.

[28] Weitzmann, M.L., 2005, Risk, Uncertainty, and Asset Pricing 'Puzzles'. Mimeo, Harvard University.

[29] Yaari, M.E. (1965): Uncertain Lifetime, Life Insurance, and the Theory of the Consumer, Review of Economic Studies, 32, 137-150. 


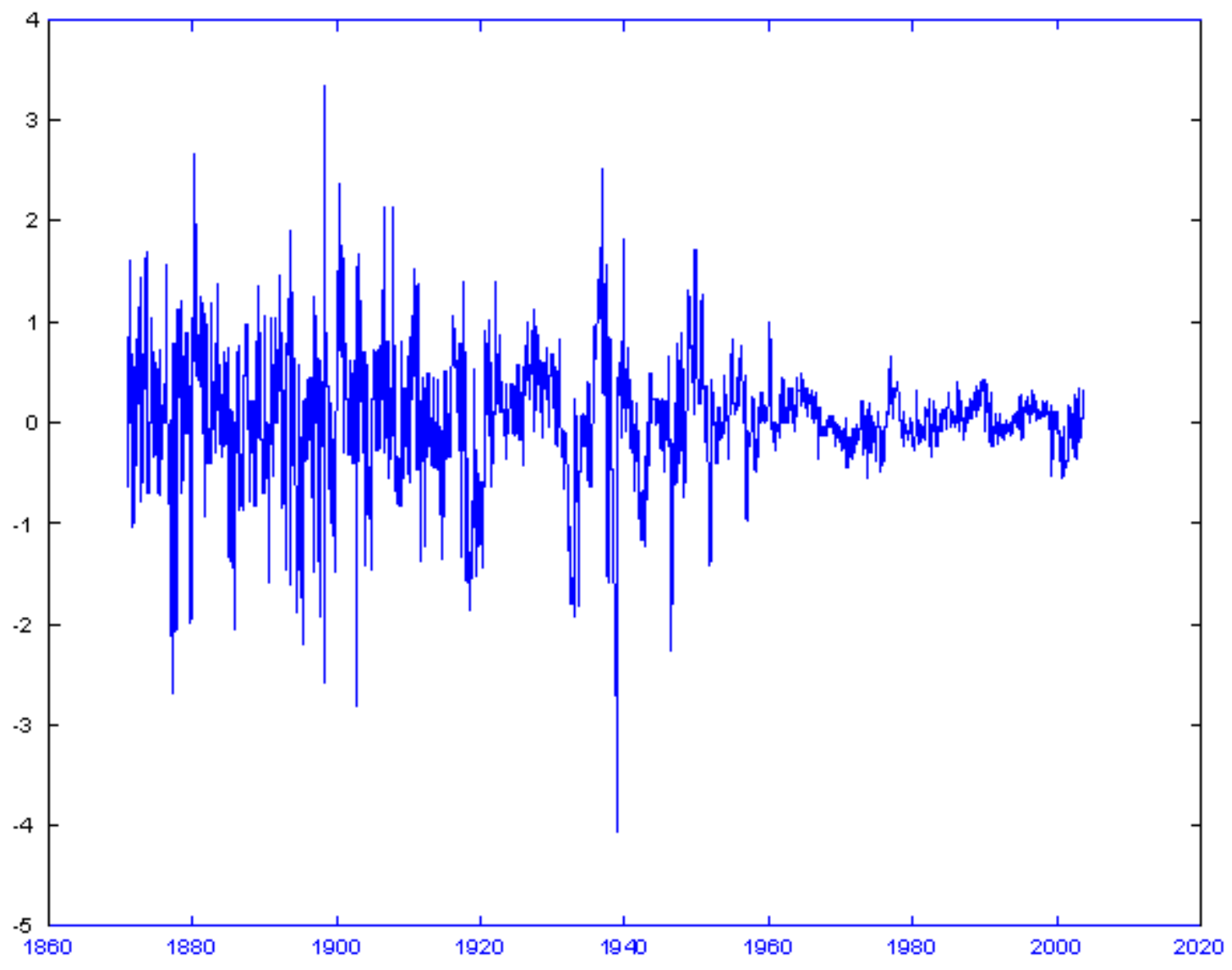

Figure 1: Monthly real dividend growth rates, 1871:3 - 2003:9 


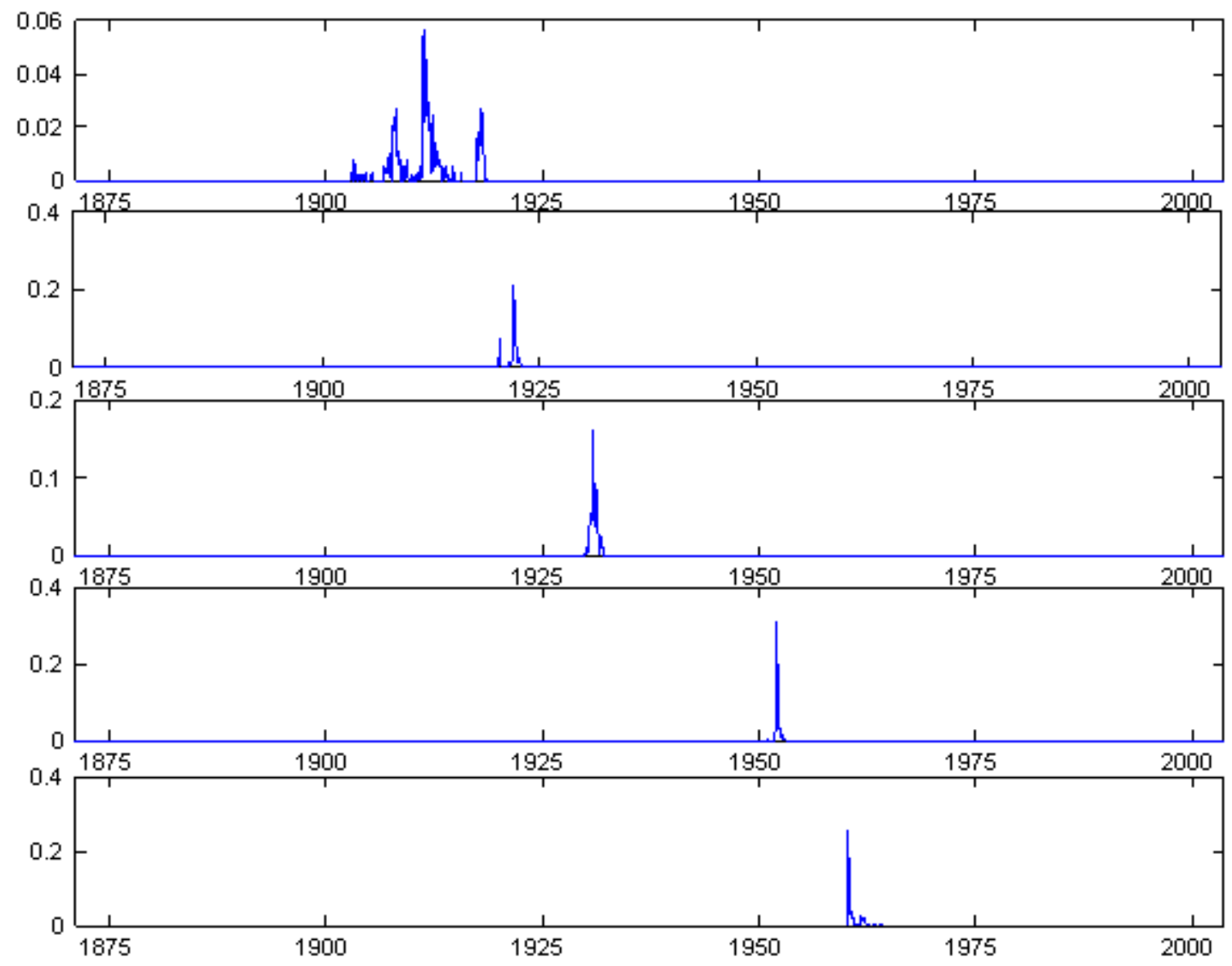

Figure 2: Posterior probability of break occurrence in the AR(1) model for the real dividend growth rate, assuming $K=5$. 

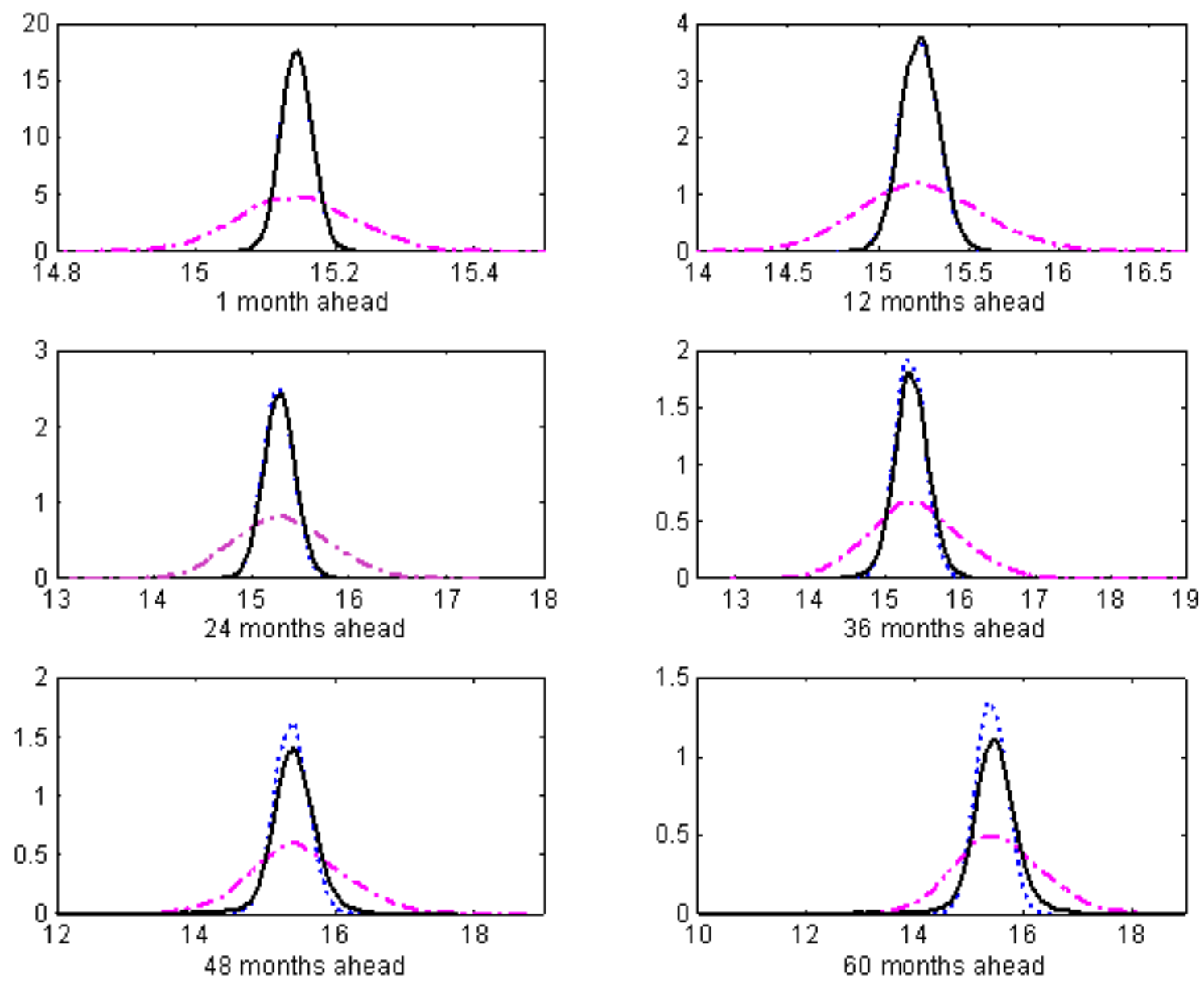

Figure 3: The graphs show the predictive distributions for the dividend series under various forecast horizons. The solid line represents the predictive density from the composite-meta model (assuming $K=5$ breaks) while the dotted line represents the predictive denstity under the last regime (19602003). Finally, the dashed/dotted line represents the predictive density from the full sample/no breaks model. 

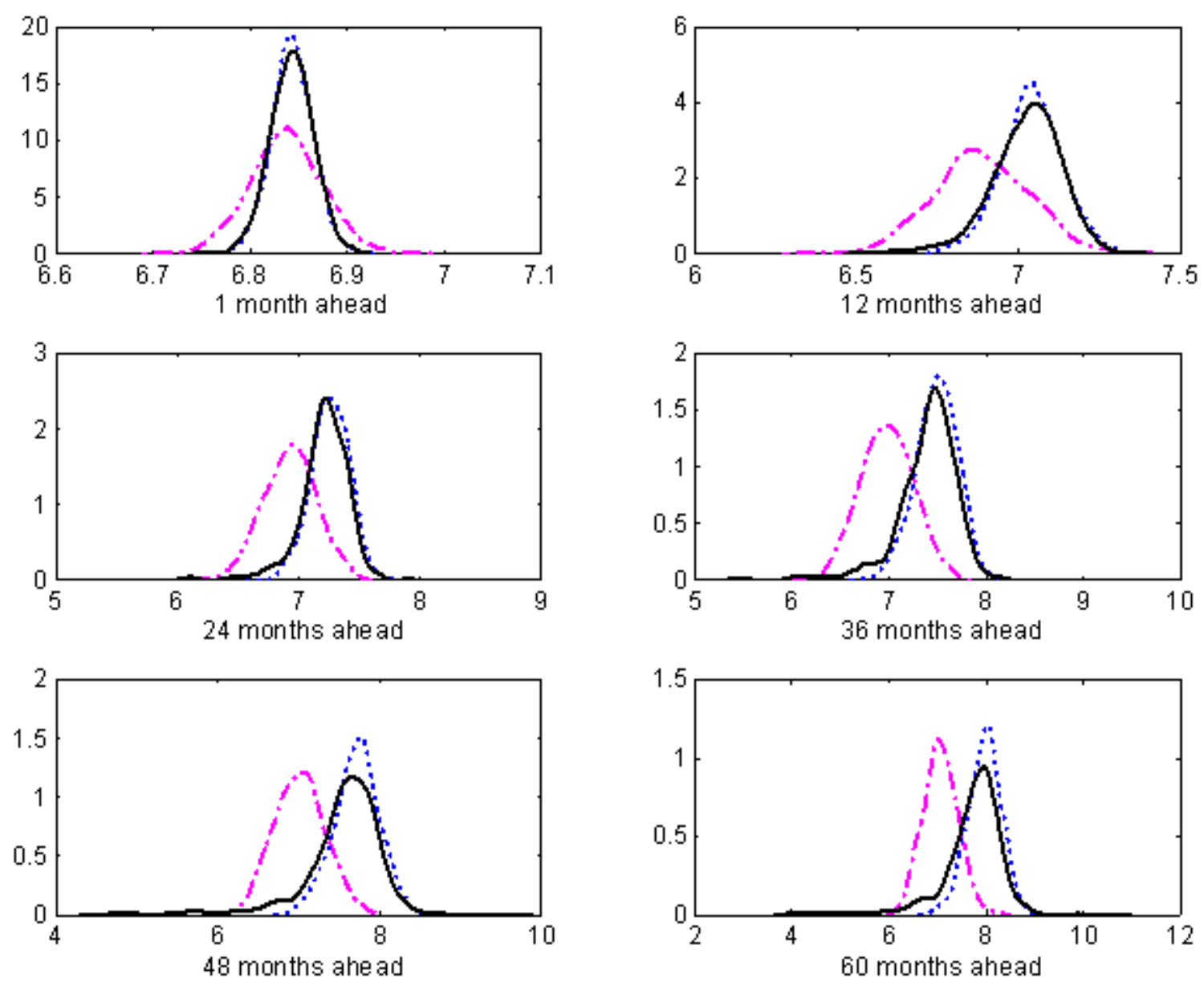

Figure 4: The graphs show the predictive distributions for the dividend series under various forecast horizons. The solid line represents the predictive density from the composite-meta model (assuming $K=5$ breaks) while the dotted line represents the predictive denstity under regime 3 (1922-1930). Finally, the dashed/dotted line represents the predictive density from the full sample/no breaks model. 

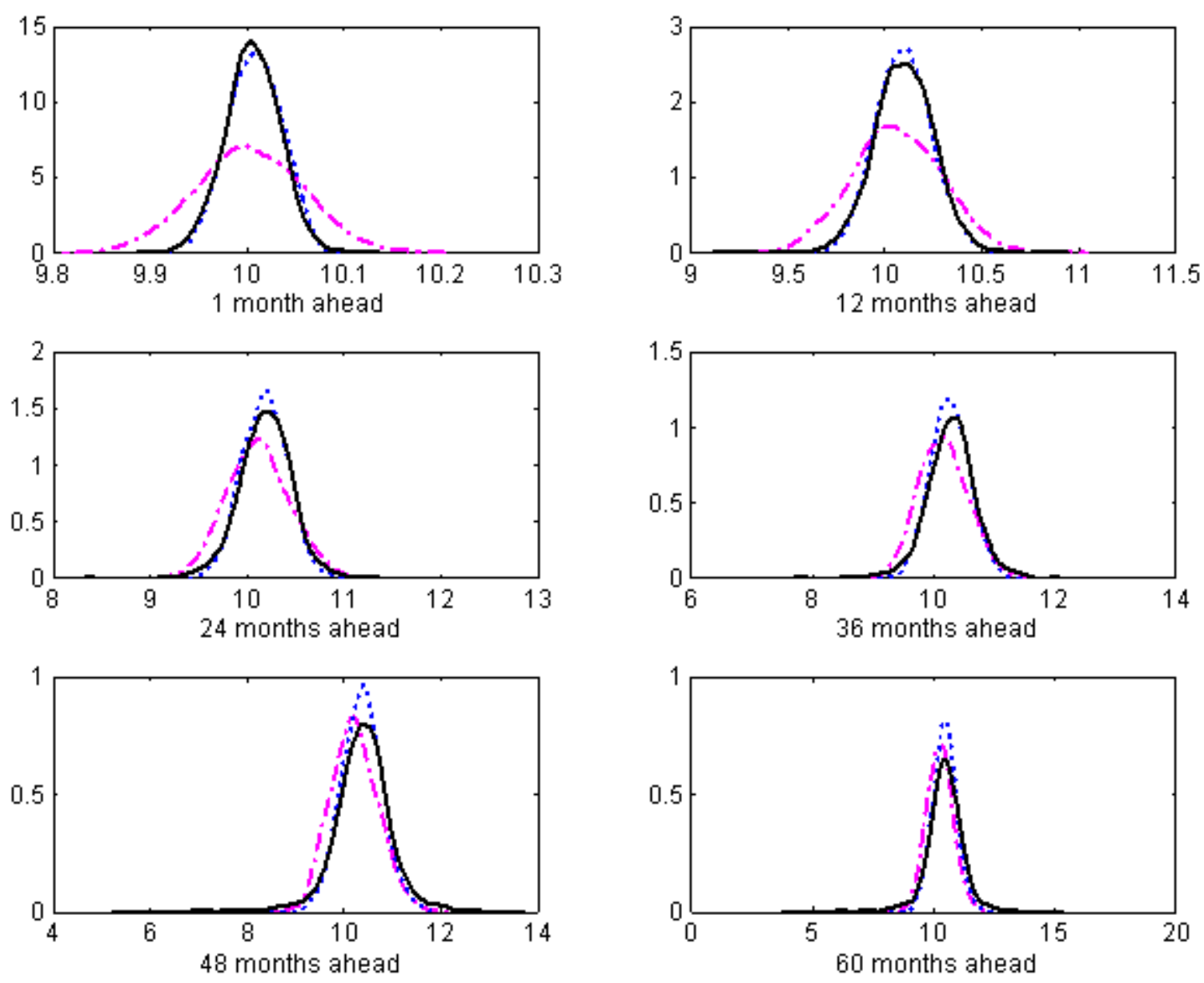

Figure 5: The graphs show the predictive distributions for the dividend series under various forecast horizons. The solid line represents the predictive density from the composite-meta model (assuming $K=5$ breaks) while the dotted line represents the predictive denstity under regime 5 (1952-1960). Finally, the dashed/dotted line represents the predictive density from the full sample/no breaks model. 


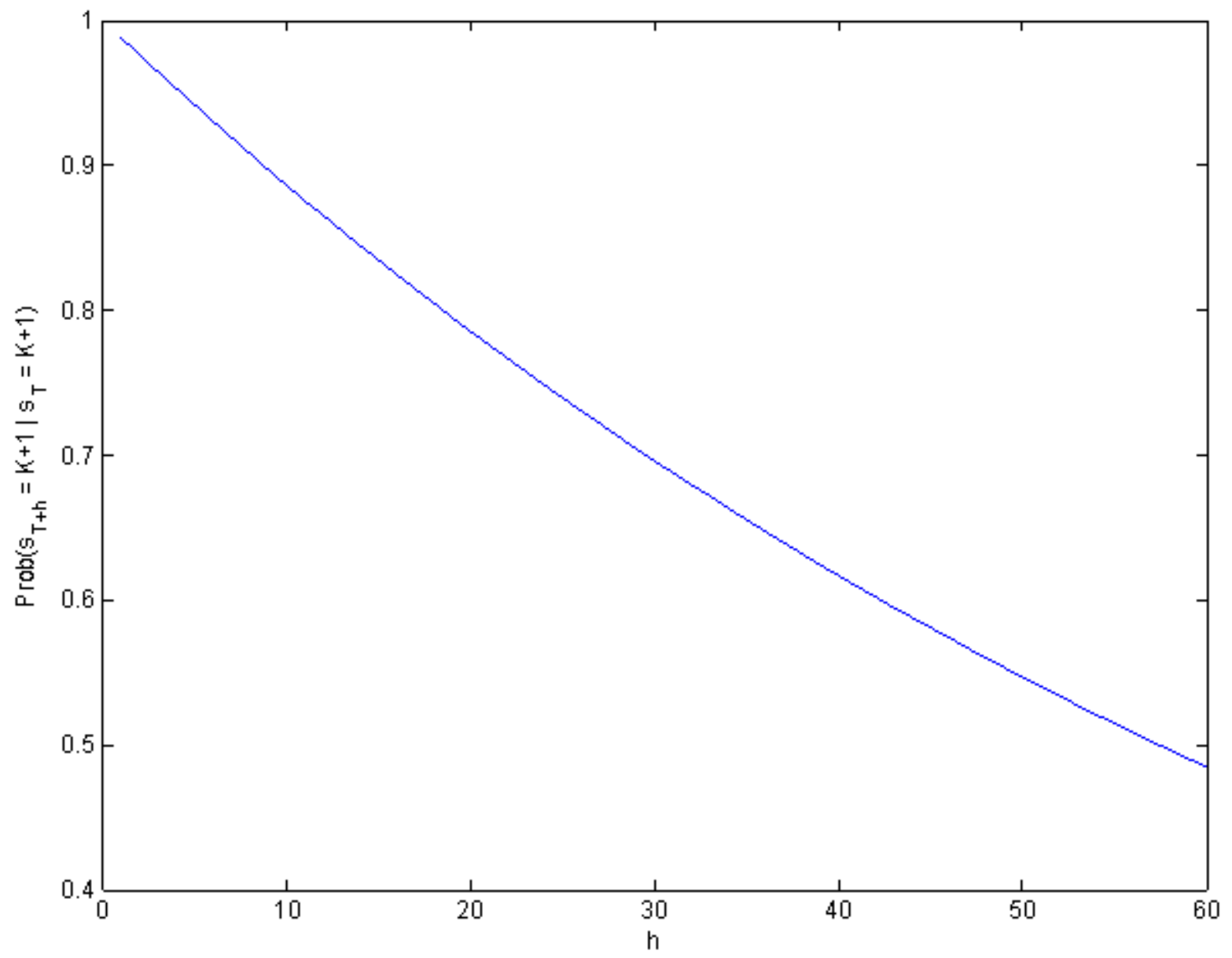

Figure 6: Posterior probability of staying in regime $K+1$ at time $T+h$, $\operatorname{Pr}\left(s_{T+h}=K+1 \mid s_{T}=K+1\right) . h$ is the forecast horizon that ranges from 1 to 60 months. 


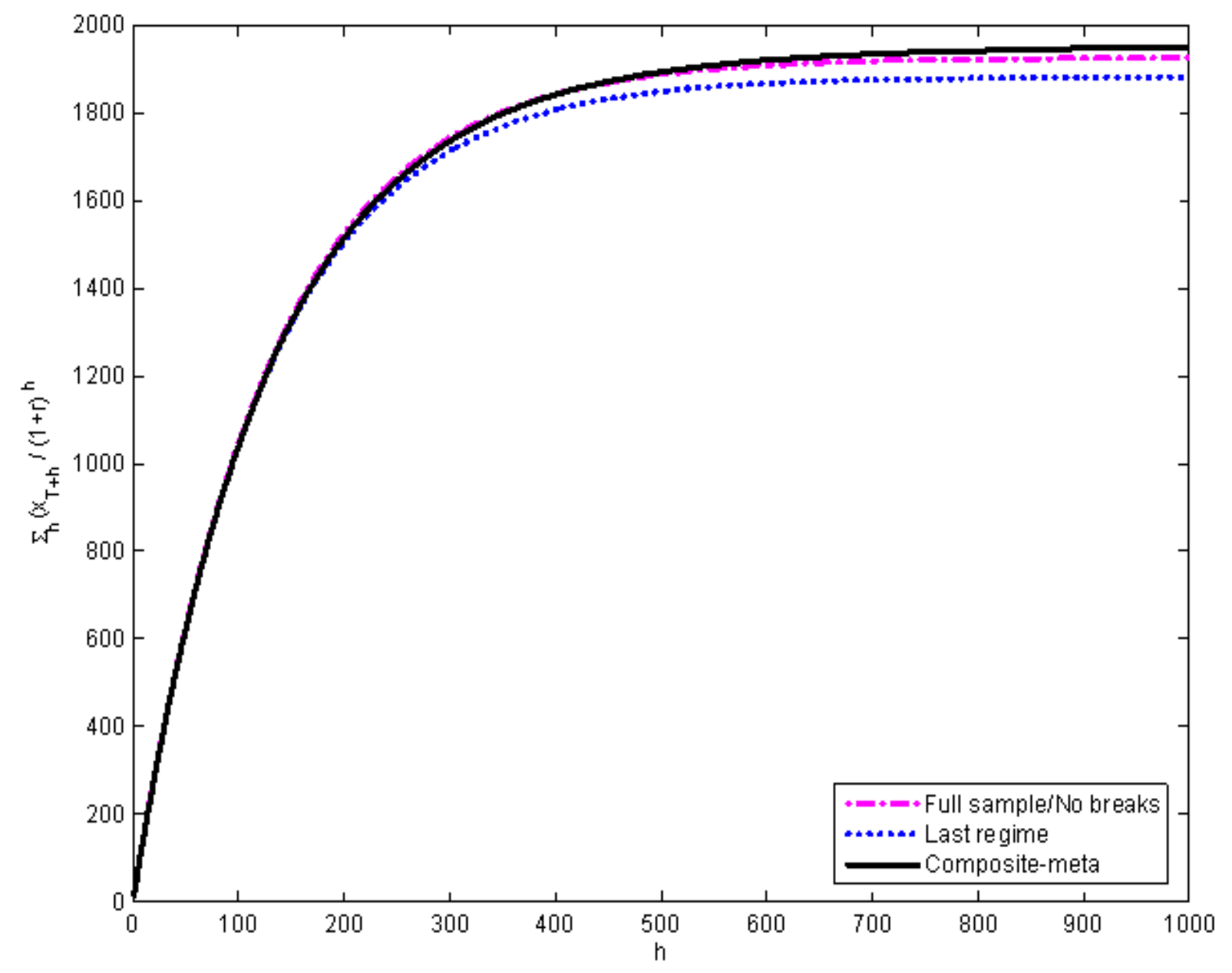

Figure 7: Cumulative discounted dividend $\sum_{j=1}^{h} \frac{x_{T+j}}{(1+r)^{j}}$ under different scenarios, where $1 \leq h \leq$ 1000 months and $r=10 \%$ per annum. The solid line represents the predictive density from the composite-meta model (assuming $K=5$ breaks) while the dotted line represents the predictive denstity under the last regime. Finally, the dashed/dotted line represents the predictive density from the full sample/no breaks model. The dividend is set at the end of sample value, $x_{T}=15.12$. 


\begin{tabular}{cccccc}
\hline \multicolumn{5}{c}{$\Delta \log \left(x_{t}\right)$} & Sample Statistics \\
\hline Mean & Std. dev. & Skewness & Kurtosis & 1st order AC & 12 th order AC \\
0.0004 & 0.0064 & -0.5133 & 7.5251 & 0.5220 & 0.0480 \\
\hline
\end{tabular}

Table 1: Sample statistics for the monthly growth rate of the real dividends, $\Delta \log \left(x_{t}\right)$. The sample period is $1871: 3-2003: 9$. 


\begin{tabular}{ccccccc}
\hline No. of breaks & Log lik.(LL) & Marginal LL & \multicolumn{5}{c}{ Break dates } \\
0 & -1298.05 & -1329.4062 & & & & \\
1 & -806.482 & -875.8323 & Feb-52 & & & \\
2 & -749.527 & -820.8898 & Sep-11 & Apr-60 & & \\
3 & -723.086 & -795.1873 & May-11 & Feb-52 & Apr-60 & \\
4 & -705.76 & -778.9393 & Jan-11 & Nov-30 & Jan-52 & Apr-60 \\
5 & -693.636 & -769.1268 & Jan-11 & Jan-22 & Nov-30 & Jan-52 \\
& & & Apr-60 & & & \\
6 & -692.465 & N.A. & Nov-17 & Jun-20 & Feb-31 & Jan-52 \\
& & & Sep-60 & Oct-60 & & \\
\hline
\end{tabular}

Table 2: Model comparison. This table shows log likelihood and marginal log likelihood values for first-order autoregressive models with different numbers of breaks. Also reported are posterior modes of the time of the breaks. 


\begin{tabular}{|c|c|c|c|c|c|c|}
\hline \multicolumn{7}{|c|}{ Parameters estimates } \\
\hline \multicolumn{7}{|c|}{ Regimes } \\
\hline & 1 & 2 & 3 & 4 & 5 & 6 \\
\hline date & $71-11$ & $11-22$ & $22-30$ & $30-52$ & $52-60$ & $60-04$ \\
\hline \multicolumn{7}{|c|}{ Constant } \\
\hline Mean & 0.071 & -0.022 & 0.155 & -0.009 & 0.030 & 0.009 \\
\hline s.e. & 0.035 & 0.044 & 0.052 & 0.033 & 0.025 & 0.006 \\
\hline \multicolumn{7}{|c|}{$\mathrm{AR}(1)$ coefficient } \\
\hline Mean & 0.372 & 0.606 & 0.398 & 0.729 & 0.621 & 0.658 \\
\hline s.e. & 0.043 & 0.073 & 0.104 & 0.042 & 0.069 & 0.032 \\
\hline \multicolumn{7}{|c|}{ Standard deviation } \\
\hline Mean & 0.781 & 0.589 & 0.310 & 0.597 & 0.270 & 0.148 \\
\hline s.e. & 0.028 & 0.039 & 0.024 & 0.027 & 0.020 & 0.005 \\
\hline \multicolumn{7}{|c|}{ Transition Probability matrix } \\
\hline Mean & 0.997 & 0.989 & 0.986 & 0.994 & 0.985 & 1 \\
\hline s.e. & 0.003 & 0.010 & 0.011 & 0.005 & 0.012 & 0 \\
\hline
\end{tabular}

Table 3: Posterior parameter estimates for the AR(1) hierarchical Hidden Markov Chain model with five break points in the monthly growth rate of real dividends. 


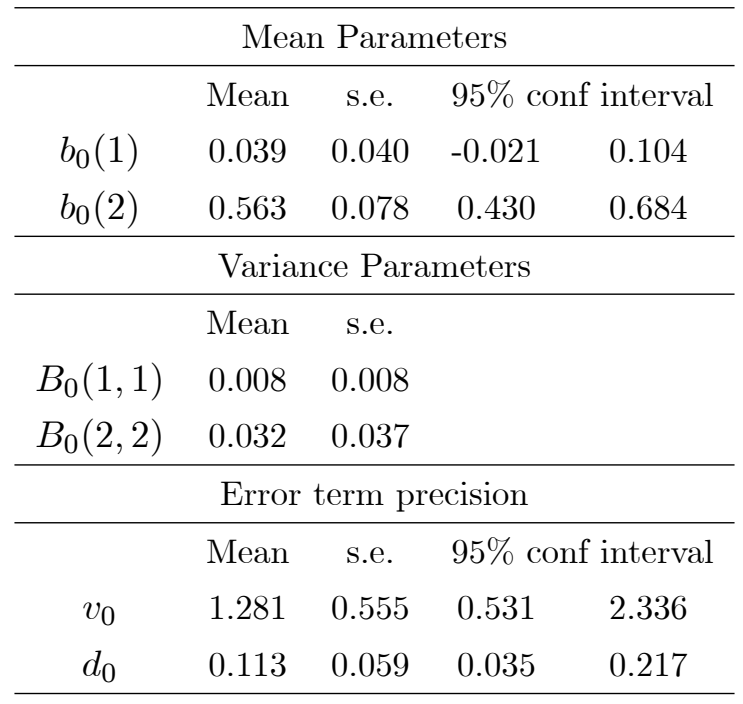

Table 4: Posterior estimates of the hyperparameters of the meta distribution for the AR(1) hierarchical Hidden Markov Chain model with five break points for the monthly growth rate of real dividends. 


\begin{tabular}{cccc}
\hline \multicolumn{4}{c}{$y_{T}$} \\
\hline Regime & Full sample/No breaks & Last regime & Composite-Meta \\
$1960-2003$ & 100 & 97.736 & 101.272 \\
$1922-1930$ & 100 & 136.427 & 120.306 \\
$1930-1952$ & 100 & 93.012 & 101.036 \\
$1952-1960$ & 100 & 104.677 & 107.656 \\
\hline
\end{tabular}

Table 5: Stock price $y_{T}$ under different scenarios when the interest rate is set at $10 \%$ per annum. 